\title{
AN ADAPTIVE FINITE ELEMENT METHOD FOR TWO-PHASE STEFAN PROBLEMS IN TWO SPACE DIMENSIONS. PART I: STABILITY AND ERROR ESTIMATES
}

\author{
R. H. NOCHETTO, M. PAOLINI, AND C. VERDI
}

\begin{abstract}
A simple and efficient adaptive local mesh refinement algorithm is devised and analyzed for two-phase Stefan problems in 2D. A typical triangulation is coarse away from the discrete interface, where discretization parameters satisfy a parabolic relation, whereas it is locally refined in the vicinity of the discrete interface so that the relation becomes hyperbolic. Several numerical tests are performed on the computed temperature to extract information about its first and second derivatives as well as to predict discrete free boundary locations. Mesh selection is based upon equidistributing pointwise interpolation errors between consecutive meshes and imposing that discrete interfaces belong to the so-called refined region. Consecutive meshes are not compatible in that they are not produced by enrichment or coarsening procedures but rather regenerated. A general theory for interpolation between noncompatible meshes is set up in $L^{p}$-based norms. The resulting scheme is stable in various Sobolev norms and necessitates fewer spatial degrees of freedom than previous practical methods on quasi-uniform meshes, namely $O\left(\tau^{-3 / 2}\right)$ as opposed to $O\left(\tau^{-2}\right)$, to achieve the same global asymptotic accuracy; here $\tau>0$ is the (uniform) time step. A rate of convergence of essentially $O\left(\tau^{1 / 2}\right)$ is derived in the natural energy spaces provided the total number of mesh changes is restricted to $O\left(\tau^{-1 / 2}\right)$, which in turn is compatible with the mesh selection procedure. An auxiliary quasi-optimal pointwise error estimate for the Laplace operator is proved as well. Numerical results illustrate the scheme's efficiency in approximating both solutions and interfaces.
\end{abstract}

\section{INTRODUCTION}

A common feature in dealing with degenerate parabolic equations is the intrinsic lack of regularity of solutions across the interfaces (or free boundaries) which, in turn, are not known in advance. For the two-phase Stefan problem, for instance, the temperature $\theta$ cannot be better than Lipschitz continuous and the enthalpy $u$ (or energy density) typically exhibits a jump discontinuity across the interface. They satisfy the PDE

$$
u_{t}-\operatorname{div}(k(\theta) \nabla \theta)=f(\theta) \quad \text { in } \Omega \times(0, T),
$$

Received September 21, 1989.

1980 Mathematics Subject Classification (1985 Revision). Primary 65N15, 65N30, 35R35.

This work was partially supported by NSF Grants DMS-8805218 and DMS-9008999, and by MPI (Fondi per la Ricerca Scientifica 40\%) and CNR (IAN, Contract 880032601 and Progetto Finalizzato "Sistemi Informatici e Calcolo Parallelo", Sottoprogetto "Calcolo Scientifico per Grandi Sistemi") of Italy. 
subject to the strongly nonlinear constitutive relation $\theta=\beta(u)$, where $\beta$ vanishes on $[0,1]$. The situation is more intricate when cusps and/or mushy regions develop. This lack of smoothness makes finite element approximations, defined on quasi-uniform meshes, perform worse than expected according to the interpolation theory. In other words, the singularity located on the interface pollutes the numerical solution everywhere. Numerical experiments for the Stefan problem indicate that the rate of convergence for temperature is never better than linear $[17,18]$. Theoretical results are even more pessimistic $[4,8,12,13,14$, $17,24]$.

Methods studied so far are not completely satisfactory in that they do not take advantage of the fact that singularities are located in a small region compared with the entire domain $\Omega$, at least whenever mushy regions do not occur. Consequently, a possible remedy is to be found in terms of a suitably designed adaptive algorithm. In fact, we would like to use a finer mesh near singularities in order to equidistribute interpolation errors but still preserve the number of degrees of freedom, and thus the computational complexity. We refer to [1, 14] for an account of the state-of-the-art on this topic along with numerous references.

In this light, the aim of this paper is to present and analyze an adaptive mesh refinement method for two-dimensional two-phase Stefan problems. We emphasize that such problems are strongly nonlinear in that singularities do not smooth out as time evolves and, more notably, they may even develop. This is a striking contrast between degenerate and purely parabolic equations. Therefore, even though various adaptive algorithms have been recently introduced for standard parabolic equations [1, 5, 14], ours appears to be the first one with a rigorous mathematical foundation for Stefan problems. We refer to [15], where a summary of some preliminary results can be found.

The finite element mesh cannot be modified in an arbitrary manner for the discrete scheme to be stable and convergent. Several tests are carried out on the computed temperature to extract information about its first and second derivatives as well as about the location of the discrete interface. Upon failure, the current mesh is discarded and a new one completely regenerated by an efficient automatic mesh generator [19]. Since the new mesh is not produced by enrichment or coarsening procedures, it happens to be noncompatible with the previous one. It is designed to be coarse away from the discrete free boundary, where the typical meshsize is $O\left(\tau^{1 / 2}\right)$, and locally refined near the interface for triangles to reach a size $O(\tau)$; hereafter, $\tau>0$ stands for the (uniform) time step. These relations, which come from elementary interpolation considerations, reflect the physical property that the Stefan problem behaves as parabolic away from the interface but possesses a first-order hyperbolic-like structure in its vicinity. On the other hand, even though the cost of generating a mesh at every single time step is asymptotically negligible compared to that of solving the associated nonlinear algebraic systems, frequent remeshing should be avoided for practical purposes. In addition, the interpolation process used to transfer infor- 
mation between consecutive meshes incorporates an error $O(\tau)$ that eventually accumulates in time. To prevent such an error from compromising accuracy, a restriction on the total number of admissible mesh changes is enforced, namely $O\left(\tau^{-1 / 2}\right)$. As the current mesh is thus to be kept fixed for a prescribed number of time iterations and discrete interfaces are supposed to lie within the so-called refined region, a further refinement is required for our strategy to succeed. This is accomplished by predicting the "small" region to be occupied by the discrete interface between consecutive mesh changes, as well as checking that it actually remains there within safe limits. The resulting scheme is stable, convergent and necessitates fewer degrees of freedom than previous methods on fixed meshes, namely $O\left(\tau^{-3 / 2}\right)$ instead of $O\left(\tau^{-2}\right)$ for well-behaved interfaces, to achieve the same global asymptotic accuracy. Moreover, it exhibits a superior performance as expressed in terms of computing time for a desired accuracy. This improvement is even more dramatic when accuracy is measured in the maximum norm.

The paper is organized as follows. In $\S 2$ we formulate the continuous and discrete problems along with the corresponding assumptions. In $\S 3$ we comment on certain heuristic aspects of our local refinement strategy, which is fully discussed in $\S 4$. In $\S 5$ and Supplement $\S S 1$ we prove several $L^{p}$-based interpolation estimates for noncompatible meshes that account for mesh change effects and play a major role in our analysis. Discrete stability in various norms is then derived in $\S 6$ and Supplement $\S S 2$. As a result, in $\S 7$ we demonstrate a rate of convergence of essentially $O\left(\tau^{1 / 2}\right)$ for both $\theta$ and $u$ in the natural energy norms, provided the total number of mesh changes is limited to $O\left(\tau^{-1 / 2}\right)$. This result agrees with previous ones $[4,17,24]$ obtained for a fixed mesh under the stronger assumption that the meshsize is $O(\tau)$. We also prove, in Supplement $\S S 3$, an auxiliary quasi-optimal pointwise error estimate for the Laplace operator, that may have some independent interest in that it extends the techniques in $[21,22,23]$ to general meshes; it is based upon a new discrete Caccioppoli estimate. To simplify the presentation, we assume that conductivity $k=1$ and that mushy regions do not occur. These interesting situations are, however, treated in some detail in Supplement $\S S 4$ along with a modification of the local mesh refinement algorithm. We conclude in $\S 8$ with several numerical experiments to illustrate the superior performance of the Adaptive Method in approximating both solutions and interfaces. Various computational issues are discussed in $\S 8$ as well.

Further numerical results and comparisons with the Fixed Mesh Method as well as implementation details will appear elsewhere [16]. They indicate a (practical) linear rate of convergence, namely $O(\tau)$, which is much better than our theoretical prediction. This topic deserves further investigation.

\section{Problem statement}

Let $\Omega \subset \mathbf{R}^{2}$ be a bounded domain with $\partial \Omega \in C^{1,1}$ and $T>0$ be fixed. The case of polygonal domains will be considered in $\S S 4.4$. Let $\beta: \mathbf{R} \rightarrow \mathbf{R}$ be 
a continuous and nondecreasing function which satisfies

$$
\begin{gathered}
0<l_{\beta} \leq \beta^{\prime}(s) \leq L_{\beta}<\infty, \quad\left|\beta^{\prime \prime}(s)\right| \leq L_{\beta^{\prime}}<\infty, \quad \text { a.e. } s \in \mathbf{R} \backslash[0,1], \\
\beta(s)=0, \quad \forall s \in[0,1] ;
\end{gathered}
$$

hence $L_{\beta}$ and $L_{\beta^{\prime}}$ are the Lipschitz constants of $\beta$ and $\beta^{\prime}$, respectively. A typical example is $\beta(s):=c_{1} s^{-}+c_{2}(s-1)^{+}$, where $c_{1}<0<c_{2}$ are fixed; this corresponds to an ideal material with constant thermal properties. Let $u_{0}$ indicate the initial enthalpy. Let $\theta_{0}:=\beta\left(u_{0}\right)$ denote the initial temperature and let $I_{0}:=\left\{x \in \Omega: \theta_{0}(x)=0\right\}$ be the initial interface. They satisfy

$$
\begin{gathered}
\theta_{0} \in W_{0}^{1, \infty}(\Omega) \cap W^{2, \infty}\left(\Omega \backslash I_{0}\right), \\
I_{0} \text { is a Lipschitz curve. }
\end{gathered}
$$

Therefore, $u_{0}$ is of bounded variation, $u_{0} \in W^{2, \infty}\left(\Omega \backslash I_{0}\right)$ and it has a jump discontinuity across $I_{0}$. In $\S S 4.2$ we will allow the initial interface $I_{0}$ to degenerate into a mushy region. The source term $f$ is also Lipschitz continuous, namely,

$$
\left|f\left(s_{1}\right)-f\left(s_{2}\right)\right| \leq L_{f}\left|s_{1}-s_{2}\right|, \quad \forall s_{1}, s_{2} \in \mathbf{R} .
$$

For the moment, the conductivity $k$ verifies $k=1$; see $\S S 4.3$ for the general case. The continuous problem then reads as follows: find $\theta$ and $u$ such that

$$
\begin{gathered}
\theta \in L^{2}\left(0, T ; H_{0}^{1}(\Omega)\right), \quad u \in L^{\infty}\left(0, T ; L^{\infty}(\Omega)\right) \cap H^{1}\left(0, T ; H^{-1}(\Omega)\right), \\
\theta(x, t)=\beta(u(x, t)), \quad \text { a.e. } x \in \Omega, \quad t \in(0, T), \\
u(\cdot, 0)=u_{0}
\end{gathered}
$$

and for a.e. $t \in(0, T)$ and all $\varphi \in H_{0}^{1}(\Omega)$ the following equation holds:

$$
\left\langle u_{t}, \varphi\right\rangle+\langle\nabla \theta, \nabla \varphi\rangle=\langle f(\theta), \varphi\rangle .
$$

Hereafter, $\langle\cdot, \cdot\rangle$ stands for the inner product on $L^{2}(\Omega)$. It is to be observed that the vanishing Dirichlet boundary condition on $\theta$ is assumed only for simplicity and, in addition, that the interface $I(t):=\{x \in \Omega: \theta(x, t)=0\}$ does not include $\partial \Omega$. Existence and uniqueness for this problem are known as well as the following further regularity results $[6,8,10]$ :

$$
\theta \in H^{1}\left(0, T ; L^{2}(\Omega)\right) \cap L^{\infty}\left(0, T ; H_{0}^{1}(\Omega)\right), \quad \Delta \theta \in L^{\infty}(0, T ; M(\Omega)),
$$

where $M(\Omega)$ stands for the space of finite regular Baire measures. In the classical situation, the free boundary motion is governed by the so-called Stefan condition

$$
\left[\nabla \theta^{+}(x, t)-\nabla \theta^{-}(x, t)\right] \cdot \nu_{x}=V(x),
$$

where $x \in I(t), \nu_{x}$ is the unit vector normal to $I(t)$ and $V(x)$ is the normal velocity of $I(t)$, both at point $x$. Consequently, if $V(x) \neq 0$, the flux 
$\nabla \theta(x, t)$ presents a jump discontinuity at $x \in I(t)$ which prevents $\theta$ from being better than globally Lipschitz continuous. Equation (2.10) may fail to hold, though, whenever cusps develop. Let $\llbracket \cdot \rrbracket$ denote the jump operator on $I$, that is $\llbracket \nabla \theta \rrbracket:=\left.\left(\nabla \theta^{+}-\nabla \theta^{-}\right)\right|_{I}$. The interface $I$ may also degenerate into a mushy region, in which case $(2.10)$ is to be replaced by the more general expression

$$
\llbracket \nabla \theta(x, t) \rrbracket \cdot \nu_{x}=\llbracket u(x, t) \rrbracket V(x), \quad x \in \partial I(t) \cap \Omega .
$$

As already said, this interesting situation is treated in some detail in $\S S 4.2$. For the moment, we suppose that mushy regions do not occur.

We now introduce the finite element approximation. Denote by $\tau:=T / N$ the time step and by $\mathscr{S}^{n}$ a partition of $\Omega$ into triangles; $\mathscr{S}^{n}$ is assumed to be weakly acute and regular uniformly in $1 \leq n \leq N$. The first condition means that for any pair of adjacent triangles the sum of the opposite angles with respect to the common side does not exceed $\pi$. Given a triangle $S \in \mathscr{S}^{n}, h_{S}$ stands for its size and verifies $\lambda \tau \leq h_{S} \leq \Lambda \tau^{1 / 2} \quad(0<\lambda, \Lambda$ fixed $)$ whereas $\rho_{S}$ denotes the diameter of the biggest ball contained in $S$. The second condition above is then equivalent to requiring $\rho_{S} \geq \sigma h_{S}$ for all $S \in \mathscr{S}^{n}$, where $0<\sigma<1$ is a fixed constant (independent of $n$ and $N$ !) [2, p. 132]. The discrete domain $\Omega^{n}:=$ $\bigcup_{S \in \mathscr{S}^{n}} S$ does not coincide with $\bar{\Omega}$. However, since the technical arguments to handle their discrepancy were introduced in [17], we omit them here by simply assuming $\Omega^{n}=\bar{\Omega}$. Nonetheless, the influence of the pollution effect due to corners will be examined in $\S \mathrm{S} 4.4$. Let $\mathbf{V}^{n} \subset H_{0}^{1}(\Omega)$ indicate the usual piecewise linear finite element space over $\mathscr{S}^{n}$ and $\Pi^{n}: C^{0}(\bar{\Omega}) \rightarrow \mathbf{V}^{n}$ the usual Lagrange interpolation operator [2, p. 94]. Finally, let $\left\{x_{j}^{n}\right\}_{j=1}^{J^{n}}$ denote the nodes of $\mathscr{S}^{n}$ and $\left\{\chi_{j}^{n}\right\}_{j=1}^{J^{n}}$ the canonical basis of $\mathbf{V}^{n}$. The discrete initial enthalpy $U^{0} \in \mathbf{V}^{1}$ is defined at a generic node $x_{j}^{1}$ of $\mathscr{S}^{0}:=\mathscr{S}^{1}$ to be

$$
U^{0}\left(x_{j}^{1}\right):=u_{0}\left(x_{j}^{1}\right), \quad \forall x_{j}^{1} \in \Omega \backslash I_{0}, \quad U^{0}\left(x_{j}^{1}\right):=1, \quad \forall x_{j}^{1} \in I_{0} .
$$

Hence, $U^{0}$ is easy to evaluate in practice. Set $\Theta^{0}:=\Pi^{1} \theta_{0} \quad\left(=\Pi^{1}\left[\beta\left(U^{0}\right)\right]\right)$. Given $U^{n-1}, \Theta^{n-1} \in \mathbf{V}^{n-1}$, the discrete scheme then reads as follows: for any $1 \leq n \leq N$ select $\mathscr{S}^{n}$ and find $U^{n}, \Theta^{n} \in \mathbf{V}^{n}$ such that

$$
\begin{gathered}
\Theta^{n}=\Pi^{n} \beta\left(U^{n}\right), \\
\widehat{U}^{n-1}:=\Pi^{n} U^{n-1}, \quad \widehat{\Theta}^{n-1}:=\Pi^{n}\left[\beta\left(U^{n-1}\right)\right], \\
\tau^{-1}\left\langle U^{n}-\widehat{U}^{n-1}, \chi\right\rangle^{n}+\left\langle\nabla \Theta^{n}, \nabla \chi\right\rangle=\left\langle f\left(\widehat{\Theta}^{n-1}\right), \chi\right\rangle^{n}, \quad \forall \chi \in \mathbf{V}^{n},
\end{gathered}
$$
where $\langle\cdot, \cdot\rangle^{n}$ is defined by

$$
\langle\varphi, \chi\rangle^{n}:=\int_{\Omega} \Pi^{n}(\varphi \chi) d x, \quad \forall \varphi, \chi \in C^{0}(\bar{\Omega}) .
$$

Note that the integral in (2.16) can be easily evaluated element-by-element via the vertex quadrature rule, which is exact for piecewise linear functions 
[2, p. 182]. The discrete interface and refined region are then defined to be $F^{n}:=\left\{x \in \Omega: \Theta^{n}(x)=0\right\}$ and $\mathscr{R}^{n}:=\bigcup\left\{S \in \mathscr{S}^{n}: h_{S}=O(\tau)\right\}$, respectively.

In view of $(2.16)$, the equation (2.15) can also be written as follows:

$$
\tau^{-1}\left\langle U^{n}-U^{n-1}, \chi\right\rangle^{n}+\left\langle\nabla \Theta^{n}, \nabla \chi\right\rangle=\left\langle f\left(\beta\left(U^{n-1}\right)\right), \chi\right\rangle^{n}, \quad \forall \chi \in \mathbf{V}^{n} \text {, }
$$

thus eliminating the definitions in (2.14). It is to be stressed that the nodes of $\mathscr{S}^{n}$ are then used for the numerical integration of piecewise linear functions defined in $\mathbf{V}^{n-1}$. The interpolation error so incurred may destroy convergence as well as stability. The mesh selection strategy of $\S 4$ will account for such an effect.

Observe that, if we first decompose the integral $\langle\nabla \varphi, \nabla \chi\rangle$ over all triangles of $\mathscr{S}^{n}$ and next integrate by parts, we get

$$
\langle\nabla \varphi, \nabla \chi\rangle=\sum_{e \in \mathscr{E}^{n}}\left\langle\left\langle\llbracket \nabla \varphi \mathbb{1}_{e} \cdot \nu_{e}, \chi\right\rangle_{e}, \quad \forall \varphi, \chi \in \mathbf{V}^{n},\right.
$$

where $\mathscr{E}^{n}:=\left\{e: e\right.$ is a side (or edge) of $S$ in $\left.\Omega, S \in \mathscr{S}^{n}\right\},\left\langle\langle\cdot, \cdot\rangle_{e}\right.$ denotes the $L^{2}$-scalar product on $e, \nu_{e}$ is the unit vector normal to $e$ and $\llbracket \cdot \mathbb{1}_{e}$ indicates the jump operator on $e$ for all $e \in \mathscr{E}^{n}$. Let $S_{j}^{n}$ denote the interior of supp $\chi_{j}^{n}$ for $1 \leq j \leq J^{n}$ and set $h_{e}:=\operatorname{length}(e)$ for $e \in \mathscr{E}^{n}$. Then (2.18) results in

$$
2\left\langle\nabla \varphi, \nabla \chi_{j}^{n}\right\rangle=\sum_{e \subset S_{j}^{n}} h_{e} \llbracket \nabla \varphi \rrbracket_{e} \cdot \nu_{e}, \quad \forall \varphi \in \mathbf{V}^{n}, \forall 1 \leq j \leq J^{n} .
$$

In view of (2.16) and (2.19), another useful relation equivalent to (2.15) reads

$$
\begin{aligned}
& \sum_{e \subset S_{j}^{n}} h_{e} \llbracket \nabla \Theta^{n} \mathbb{1}_{e} \cdot \nu_{e} \\
& \quad=\frac{2}{3} \operatorname{meas}\left(S_{j}^{n}\right)\left(f\left(\widehat{\Theta}^{n-1}\left(x_{j}^{n}\right)\right)-\tau^{-1}\left(U^{n}-\widehat{U}^{n-1}\right)\left(x_{j}^{n}\right)\right) .
\end{aligned}
$$

From now on, $C>0$ will denote a constant independent of $\tau$ but not necessarily the same at each occurrence. Moreover, $C$ may depend on the given data as well as on the various constants to be introduced in $\S 4$. The notation $\cdot=O\left(\tau^{\gamma}\right)$ will be often used instead of $\leq \leq C \tau^{\gamma}$. As usual, $|x|$ will stand for any norm of $x \in \mathbf{R}^{2}$.

\section{HeURISTIC GUIDELINES}

We now give a heuristic motivation for the local refinement strategy of $\S 4$. We first consider the following 1-D problem discretized only in time:

$$
U-\tau \beta(U)_{x x}=u_{0} \quad \text { in }(-1,1),
$$

where $\beta(s):=(s-1)^{+}-s^{-}, V:=\gamma_{2}-\gamma_{1}>0$ and $u_{0}(x):=\gamma_{2}\left(e^{V x}-1\right) / V+1$ if $x>0, u_{0}(x):=\gamma_{1}\left(e^{V x}-1\right) / V$ if $x<0$. Since $u(x, \tau)=u_{0}(x+V \tau)$, the interface $I(t)$, initially at 0 , reaches $-V \tau$ at time $\tau$. Let $\delta$ denote the 
position of the discrete-time interface $F$. It is not difficult, but tedious, to see that $(\Theta:=\beta(U))$

$$
\begin{gathered}
\|\Theta-\theta(\tau)\|_{L^{\infty}((-1,1))}=O(\tau) \geq V^{2} \tau \\
\delta=-V \tau+O\left(\tau^{3 / 2}\right), \\
\Theta_{x}\left(\delta^{-}\right)=\Theta_{x}\left(\delta^{+}\right)=\gamma_{1}+O\left(\tau^{1 / 2}\right) \quad \text { (smearing effect!), } \\
\Theta_{x}(0)=\gamma_{2}+O\left(\tau^{1 / 2}\right), \\
\Theta_{x x}(x)=\tau^{-1}+O(1), \quad \forall \delta<x<0 .
\end{gathered}
$$

On the other hand, suppose $(-1,1)$ is partitioned into equal intervals of size $h$. Then, in view of the shape of $\theta(\tau):=\beta(u(\tau))$ and (3.6), the pointwise interpolation errors in space satisfy

$$
\begin{gathered}
\left\|\theta(\tau)-\Pi^{1} \theta(\tau)\right\|_{L^{\infty}((-1,1))} \leq V h / 4+O\left(h^{2}\right), \\
\left\|\Theta-\Pi^{1} \Theta\right\|_{L_{((-1,1))}^{\infty}} \leq h^{2} \tau^{-1} / 8+O\left(h^{2}\right) .
\end{gathered}
$$

What we learn from this relevant example can be expressed as follows. Since we expect to deal with Lipschitz continuous temperatures, the local meshsize $h_{S}$ near $F$ and interface velocity $V_{S}$ should verify $h_{S} \approx V_{S} \tau$ to balance the interpolation errors in space (3.7) with the truncation error in time (3.2). In addition, no condition similar to $(2.10)$ is valid for the semidiscrete problem at $F$, even though the free boundary moves correctly. To retrieve the proper jump condition, however, we just have to move a distance $\delta$ backwards along the normal to $F$ because, by virtue of (3.4) and (3.5),

$$
\Theta_{x}(0)-\Theta_{x}\left(\delta^{-}\right)=V+O\left(\tau^{1 / 2}\right),
$$

or, equivalently, $V=\int_{\delta}^{0} \Theta_{x x}(s) d s+O\left(\tau^{1 / 2}\right)$. Consequently, an overrefinement near the interface is extremely dangerous in that we may lose information on the interface velocity without gaining accuracy and, as a result, we might be in trouble to predict its future position. We thus realize that enforcing these two observations would require a stepwise control of the relation $h_{S} \approx V_{S} \tau$, where $V_{S}$ could be determined by means of (3.8) with $\delta$ being replaced by $h_{S}$. On the other hand, there is an interval $O(\tau)$-long behind $F$, namely $(\delta, 0)$, on which second derivatives are $O\left(\tau^{-1}\right)$.

Away from the interface $I$, problem (1.1) is strictly parabolic, namely,

$$
c(\theta) \theta_{t}-\Delta \theta=f(\theta)
$$

which is a mildly nonlinear heat equation; $c(s):=1 / \beta^{\prime}\left(\beta^{-1}(s)\right)$ for all $s \in$ $\mathbf{R} \backslash[0,1]$. Hence the discretization parameters should verify the usual parabolic constraint $h_{S}=O\left(\tau^{1 / 2}\right)$.

These two distinct behaviors, rephrased here in terms of local regularity, must be reflected in the local refinement algorithm, for instance, as illustrated in $\S 4$. 
Let us now explore some heuristic properties of the fully discrete scheme (2.15). Near the discrete interfaces, where the best we can say is $\left|\left(U^{n}-\widehat{U}^{n-1}\right)\left(x_{j}^{n}\right)\right| \leq C,(2.20)$ results in $\left|\sum_{e \subset S_{j}^{n}} h_{e} \llbracket \nabla \Theta^{n} \mathbb{1}_{e} \cdot \nu_{e}\right| \leq C \tau^{-1}$ meas $\left(S_{j}^{n}\right)$. Hence, except for a very unlikely cancellation in the above summation, we can expect discrete second derivatives $D_{e}$ to verify

$$
D_{e}:=h_{e}^{-1} \mid\left[\mathbb{[} \nabla \Theta^{n} \mathbb{1}_{e} \mid \leq C \tau^{-1} h_{e}^{-2} \text { meas }\left(S_{j}^{n}\right) \leq C \tau^{-1} .\right.
$$

This is consistent with (3.6). Moreover, away from the discrete free boundaries, we can expect $\left|\left(U^{n}-\widehat{U}^{n-1}\right)\left(x_{j}^{n}\right)\right| \leq C\left|\left(\Theta^{n}-\widehat{\Theta}^{n-1}\right)\left(x_{j}^{n}\right)\right| \leq C \tau$, because of (2.1), (2.13), (2.14) and the strict parabolicity of (3.9). Thus $\left|\sum_{e \subset S_{j}^{n}} h_{e} \llbracket \nabla \Theta^{n} \mathbb{1}_{e} \cdot \nu_{e}\right| \leq$ $C$ meas $\left(S_{j}^{n}\right)$. Therefore, arguing as before, we conclude that

$$
D_{e} \leq C h_{e}^{-2} \text { meas }\left(S_{j}^{n}\right) \leq C,
$$

for all $e$ in the parabolic region. The heuristic observations (3.10) and (3.11) regarding $D_{e}$, as well as the smearing effect (3.4), were confirmed by 2-D numerical experimentation. It also revealed the validity of the following $L^{1}$-type a priori estimate:

$$
\sum_{e \in \mathscr{E}^{n}} h_{e}^{2} D_{e} \leq C
$$

This property is a discrete analogue of (2.9), i.e., $\Delta \theta \in L^{\infty}(0, T ; M(\Omega))$. It is still in good agreement with numerical evidence. Indeed, actual computations show the occurrence of a strip $O(\tau)$-wide behind the discrete interface $F^{n}$ where $D_{e}=O\left(\tau^{-1}\right)$, which in turn is consistent with (3.6) and (3.10). In this case, since the local meshsize near $F^{n}$ should be $h_{e}=O(\tau),(3.12)$ imposes a severe regularity restriction on the interface, namely,

$$
\text { length }\left(F^{n}\right) \leq \sum_{e \in \mathscr{E}^{n}: e \cap F^{n} \neq \varnothing} h_{e} \leq C \sum_{e \in \mathscr{E}^{n}} h_{e}^{2} D_{e} \leq C .
$$

Such a condition is quite reasonable for practical purposes but is not known to hold in a general setting. We stress that without some kind of additional regularity it is probably hopeless to improve upon the Fixed Mesh Method [4, $8,12,13,17,24]$. In this light, (3.12) is always assumed at the mesh changes and used in $\S \S 6,7, \mathbf{S} 4$, though it constitutes a limitation of the Adaptive Method. It is however partially justified by Lemma 6.5 which, being implicitly guaranteed by the scheme, combines with (2.20) to yield

$$
\sum_{j=1}^{J^{n}}\left|\sum_{e \subset S_{j}^{n}} h_{e} \llbracket \nabla \Theta^{n} \mathbb{1}_{e} \cdot \nu_{e}\right| \leq 2 \int_{\Omega} \Pi^{n}\left(\left|f\left(\hat{\Theta}^{n-1}\right)\right|+\tau^{-1}\left|U^{n}-\widehat{U}^{n-1}\right|\right) \leq C,
$$

for all time steps $n$ between consecutive mesh changes. We then see that only a cancellation in the above summation could lead to a bound weaker than (3.12). This seems to be unlikely for locally smooth interfaces, as well as for cusps, 
because of their local character. At the same time, Lemma 5.10 shows that (3.12) is preserved for $\hat{\boldsymbol{\Theta}}^{n}$. This somehow explains the fact that (3.12) was never violated in our numerical experiments. Designing an algorithm for which (3.12) is implicitly guaranteed constitutes a challenging open problem, though. Note that mushy regions may occur as long as their boundaries are also wellbehaved. On the other hand, regarding first derivatives, the following $L^{2}$-type a priori estimate is implicitly guaranteed (see (6.6)):

$$
\left.\sum_{S \in \mathscr{S}^{n}} h_{S}^{2}\left|\nabla \Theta^{n}\right|_{S}\right|^{2} \leq C .
$$

We finally comment upon the effect of interpolation between noncompatible meshes. Let $\zeta: \mathbf{R} \rightarrow \mathbf{R}$ be sufficiently smooth and $S \in \mathscr{S}^{n}$ be a generic element. Proceed then formally, as if $\Theta^{n-1}$ were smooth, to deduce that

$$
\begin{aligned}
\| \zeta\left(\Theta^{n-1}\right)- & \Pi^{n}\left[\zeta\left(\Theta^{n-1}\right)\right] \|_{L^{\infty}(S)} \\
& \leq C h_{S}^{2}\left(\left\|D^{2} \Theta^{n-1}\right\|_{L^{\infty}(S)}+\left\|D \Theta^{n-1}\right\|_{L^{\infty}(S)}^{2}\right),
\end{aligned}
$$

where $D$ and $D^{2}$ denote discrete first and second derivatives, respectively. In $\S 5$ we give proper justification for (3.16). Since we want this interpolation error to be $O(\tau)$, the new local meshsize should satisfy

$$
h_{S} \leq \tau^{1 / 2} \min \left(\mu_{1}\left\|D^{2} \Theta^{n-1}\right\|_{L^{\infty}(S)}^{-1 / 2}, \mu_{2}\left\|D \Theta^{n-1}\right\|_{L^{\infty}(S)}^{-1}\right) .
$$

This in turn allows second derivatives to blow up without violating $h_{S} \geq$ $\lambda \tau$ as long as $\left\|D^{2} \Theta^{n-1}\right\|_{L^{\infty}(S)} \leq\left(\mu_{1} / \lambda\right)^{2} \tau^{-1}$, which is consistent with (3.10). First derivatives may also degenerate without violating $h_{S} \geq \lambda \tau$ provided $\left\|D \Theta^{n-1}\right\|_{L^{\infty}(S)} \leq\left(\mu_{2} / \lambda\right) \tau^{-1 / 2}$. Such a degeneracy is expected only whenever cusps develop, this being a local phenomenon. In addition, having control of quadrature errors introduced by $(2.16)$ leads to restrictions on triangle diameters wherever $\left\|D \Theta^{n-1}\right\|_{L^{\infty}(S)}$ exceeds a certain tolerance; this is accomplished via (3.17) as well.

On the other hand, for all $S \in \mathscr{S}^{n-1}$ intersecting the discrete interface $F^{n-1}$ we have $\left\{x \in S: 0 \leq U^{n-1}(x) \leq 1\right\} \neq \varnothing$. For sample problems having a nicely behaved continuous free boundary and verifying a nondegeneracy property, numerical experiments indicate that $U^{n-1}$ may vary from 0 to 1 within one single element. Consequently, even a slight perturbation of triangles $S$ traversed by $F^{n-1}$ would produce an error $\left\|U^{n-1}-\Pi^{n} U^{n-1}\right\|_{L^{\infty}(S)}=O(1)$ and a subsequent optimal lower bound $\left\|U^{n-1}-\Pi^{n} U^{n-1}\right\|_{L^{1}(\Omega)} \geq C \tau$, which could be attained provided length $\left(F^{n-1}\right)=O(1)$. This property of $F^{n-1}$ is not enough, however, to ensure the validity of another crucial interpolation estimate (Lemma 5.6), namely $\left\|\nabla\left(\Theta^{n-1}-\widehat{\Theta}^{n-1}\right)\right\|_{L^{2}(\Omega)} \leq C \tau^{1 / 2}$, unless $\nabla \Theta^{n-1}$ is bounded on $F^{n-1}$; strong stability would thus break down too (Lemma 6.4). Since such a further 
constraint on $\nabla \Theta^{n-1}$ rules out the formation of cusps, we should not modify triangles crossed by $F^{n-1}$. For computational purposes, it is always preferable not to impose this constraint, which is feasible whenever the interface is "smooth"; see $\S \mathrm{S} 4.1$.

\section{LOCAL REFINEMENT STRATEGY}

The aim of this section is to describe the relation between two successive and noncompatible meshes, say $\mathscr{S}^{n-1}$ and $\mathscr{S}^{n}$, along with the necessary numerical tests to be performed on $\Theta^{n-1}$. The initial mesh $\mathscr{S}^{0}\left(=\mathscr{S}^{1}\right)$ is constructed along the same lines with $\theta_{0}$ in place of $\Theta^{n-1}$. Since no confusion is possible, for simplicity we remove the superscripts and use the following notation: $\mathscr{S}:=$ $\mathscr{S}^{n-1}, \widehat{\mathscr{S}}:=\mathscr{S}^{n}, \mathscr{E}:=\mathscr{E}^{n-1}, \hat{\mathscr{E}}:=\mathscr{E}^{n}, \mathscr{R}:=\mathscr{R}^{n-1}, \widehat{\mathscr{R}}:=\mathscr{R}^{n}, \Pi:=$ $\Pi^{n-1}, \widehat{\Pi}:=\Pi^{n}, U:=U^{n-1}, \widehat{U}:=\widehat{U}^{n-1}(=\widehat{\Pi} U), \Theta:=\Theta^{n-1}(=\Pi \beta(U))$, $\widehat{\Theta}:=\widehat{\Pi} \beta(U)$ and $F:=F^{n-1}$, for $2 \leq n \leq N$. In $\S 4.1$ we introduce three local parameters that represent the expected value of local meshsize. We discuss the mesh selection algorithm in $\S 4.2$ and conclude with several comments in $\S 4.3$.

4.1. Local mesh parameters. Note that $\left.\nabla \Theta\right|_{S},\left.\nabla U\right|_{S} \in\left[P^{0}(S)\right]^{2}$ for all $S \in \mathscr{S}$. Set $d_{S}:=\left.|\nabla \Theta|_{S}\left|, D_{S}:=\right| \nabla U\right|_{S} \mid, \delta_{S}:=h_{S} d_{S}$ for all $S \in \mathscr{S}$ and

$$
\delta_{e}:=|\nabla \Theta|_{S_{1}}-\left.\nabla \Theta\right|_{S_{2}}|=| \mathbb{L} \nabla \Theta \mathbb{1}_{e} \mid, \quad D_{e}:=\frac{\delta_{e}}{h_{e}}, \quad \forall e \in \mathscr{E},
$$

where $S_{1}, S_{2} \in \mathscr{S}$ are so that $e=S_{1} \cap S_{2}$; set $\mathscr{A}_{e}:=\left\{S_{1}, S_{2}\right\}$. Note that these quantities are easy to evaluate in practice. We then introduce the following local parameters:

$$
\begin{array}{ll}
\hat{h}_{e}:=\mu_{1} \frac{\tau^{1 / 2}}{D_{e}^{1 / 2}}, & \forall e \in \mathscr{E} \backslash \mathscr{E}_{F}, \\
\hat{h}_{S}:=\mu_{2} \frac{\tau^{1 / 2}}{d_{S}}, & \forall S \in \mathscr{S} \backslash \mathscr{S}_{F},
\end{array}
$$

where $\mathscr{S}_{F}:=\{S \in \mathscr{S}: S \cap F \neq \varnothing\}, \mathscr{E}_{F}:=\left\{e \in \mathscr{E}: e \subset \partial S, S \in \mathscr{S}_{F}\right\}$ and $\mathscr{F}:=\bigcup_{S \in \mathscr{S}_{F}} S$. Here, $\mu_{1}, \mu_{2}>0$ are arbitrary constants which, in practice, result from computational considerations as well as specific properties of the problem at hand; the same comment applies to $\lambda, \Lambda$. The two local parameters above account for the effect of interpolation between noncompatible meshes, as motivated by (3.17). In case they violate the constraint $\hat{h}_{e}, \hat{h}_{s} \geq \lambda \tau$, we say that discrete derivatives are badly-behaved. This situation will require special care, even though it was never observed in practice. To this end, we set $\mathscr{S}_{B}:=\left\{S \in \mathscr{S} \backslash \mathscr{S}_{F}: \min _{e \in \mathscr{E} \backslash \mathscr{E}_{F}: e \subset \partial S}\left(\hat{h}_{S}, \hat{h}_{e}\right)<\lambda \tau\right\}, \mathscr{E}_{B}:=\{e \in$ $\left.\mathscr{E} \backslash \mathscr{C}_{F}: e \subset \partial S, S \in \mathscr{S}_{B}\right\}, \mathscr{B}:=\bigcup_{S \in \mathscr{S}_{B}} S$ and $\mathscr{S}_{0}:=\mathscr{S} \backslash\left(\mathscr{S}_{F} \cup \mathscr{S}_{B}\right), \mathscr{E}_{0}:=$ $\mathscr{E} \backslash\left(\mathscr{E}_{F} \cup \mathscr{E}_{B}\right), \Omega_{0}:=\Omega \backslash(\mathscr{F} \cup \mathscr{B})$.

We now focus our attention on the local meshsize near $F$ for problems without mushy regions. Inspired by (3.8) and subsequent heuristics $h_{S} \approx V_{S} \tau$, 
for each $S \in \mathscr{S}$ crossed by the discrete interface $F$, we compute a discrete interface velocity $V_{S}$ using a suitable discretization of condition (2.10), namely,

$$
V_{S}:=\left(\left.\nabla \Theta\right|_{S_{1}}-\left.\nabla \Theta\right|_{S_{2}}\right) \cdot \nu,
$$

where $S_{1}, S_{2} \in \mathscr{S}$ belong to each phase, are displayed on the direction $\nu$ (unit vector normal to $F$ ) and satisfy $\operatorname{dist}\left(S_{i}, S\right) \geq h_{S}(i=1,2)$. We next consider a cone $\mathscr{C}_{S}$ of axis $\nu$, vertex at $S$, opening $\pi / 2$ and height $\mu_{3} V_{S} \tau^{1 / 2}$ as being the region most likely to contain the evolution of $F_{S}:=S \cap F$ for at least $O\left(\tau^{-1 / 2}\right)$ time steps. The local parameter associated with the interface is defined by

$$
\hat{h}_{F_{S}}:=\tau \min \left\{\max \left(\lambda, V_{S}\right), M\right\} .
$$

The above two new constants $\mu_{3}$ and $M$ are arbitrary at this stage. The same rules of selection as for the previous four constants apply; $\mu_{3}$ may depend on $n$.

The union of all cones $\mathscr{C}_{S}$ constitutes the predicted refined region $\mathscr{R}$ whose width is $O\left(\tau^{1 / 2}\right)$. Note that $\mathscr{R} \subset \widehat{\mathscr{R}}$. The size of $\mathscr{R}$ enables discrete interfaces to remain within $\widehat{\mathscr{R}}$ for at least $O\left(\tau^{-1 / 2}\right)$ time steps, as desired.

4.2. Mesh selection algorithm. As already said, the initial mesh $\mathscr{S}^{1}$ is built with the required pointwise information extracted from $\theta_{0}$. Assuming now that we have a mesh $\mathscr{S}$, we would like to discuss the three tests to be performed on the computed solution $\Theta$ to either accept or discard $\mathscr{S}$.

The first test consists of checking whether the discrete interface $F$ is within the refined region $\mathscr{R}$ or not. In the event $F$ escapes from $\mathscr{R}$, we say that the test has failed.

The second test ascertains that interpolation errors are still equidistributed correctly:

$$
h_{e} \leq \mu_{1}^{*} \hat{h}_{e}, \quad \forall e \in \mathscr{E}_{0}, \quad h_{S} \leq \mu_{2}^{*} \hat{h}_{S}, \quad \forall S \in \mathscr{S}_{0} ;
$$

here $\mu_{1}^{*}, \mu_{2}^{*}>1$ are suitable constants. This rules out the possibility of an excessive refinement induced by large discrete derivatives. However, the new local meshsize might be much smaller than the current one, if influenced by the new refined region $\widehat{\mathscr{R}}$. The example in $\S 8.3$, for instance, makes (4.6) fail; see also $[16, \S 7]$.

Sometimes the interface velocity may vary substantially during an $O\left(\tau^{1 / 2}\right)$ period of time so as to make (4.5) inadequate. More specifically, the local truncation error (3.2) would not be properly reflected in the local meshsize and also, in case the current meshsize becomes too small, the computation of $V_{S}$ via (4.4) might be inaccurate because of the smearing effect (3.4). In addition, the fact that triangles of $\mathscr{S}_{F}$ are fixed and new nearby elements might have a much smaller size would create serious programming difficulties in specifying $\widehat{\mathscr{R}}$. To prevent that from happening, a third test is enforced, namely,

$$
\mu_{3}^{-} \hat{h}_{F_{S}} \leq h_{S} \leq \mu_{3}^{+} \hat{h}_{F_{S}}, \quad \forall S \in \mathscr{S}_{F},
$$


where $\mu_{3}^{-}<1<\mu_{3}^{+}$are suitable constants. A relevant example that makes (4.7) fail is that in $[16, \S 7.3]$.

If any one of the above tests fails, then the current mesh $\mathscr{S}$ is rejected as well as the solution $\{\Theta, U\}$, which is overwritten with the previously computed solution. A new graded mesh $\widehat{\mathscr{S}}$ with the following properties is then generated. To preserve the constraint $h_{\widehat{S}} \geq \lambda \tau$, we must keep $\mathscr{S}_{B}$ fixed because discrete derivatives are badly-behaved. In addition, in accord with the last heuristic observation of $\S 3$, we must not modify $\mathscr{S}_{F}$. Hence,

$$
S \in \widehat{\mathscr{S}}, \quad \forall S \in \mathscr{S}_{B} \cup \mathscr{S}_{F},
$$

is the first restriction on $\widehat{\mathscr{S}}$. The second one reads

$$
\lambda \tau \leq h_{\widehat{S}} \leq \min _{\substack{S^{\prime} \in \mathscr{S}_{F}: \mathscr{E}_{S^{\prime}} \cap \widehat{S} \neq \varnothing \\ S \in \mathscr{S}_{0}, e \in \mathscr{E}_{0}: S \cap \hat{S} \neq \varnothing, e \subset \partial S}}\left(\Lambda \tau^{1 / 2}, \hat{h}_{F_{S^{\prime}}}, \hat{h}_{e}, \hat{h}_{S}\right), \quad \forall \widehat{S} \in \widehat{\mathscr{S}} .
$$

This accounts for both the equidistribution of pointwise interpolation errors (3.17) and the definition of refined region $\widehat{\mathscr{R}}$. The effective implementation of (4.9) will be discussed in $\S 8.1$; see also $[16, \S 6]$.

4.3. Further properties. The information about discrete derivatives could be extracted from $U$ rather than $\theta$ because, in view of (2.1) and (2.13), they are equivalent on $\Omega_{0}$. In fact, for all $e \in \mathscr{E}_{0}$ and $S \in \mathscr{S}_{0}$, we have

$$
\begin{gathered}
h_{e}^{-1}|\nabla U|_{S_{1}}-\left.\nabla U\right|_{S_{2}} \mid \leq C\left(l_{\beta}^{-1} D_{e}+L_{\beta^{\prime}} l_{\beta}^{-3}\left(d_{S_{1}}^{2}+d_{S_{2}}^{2}\right)\right), \quad S_{i} \in \mathscr{A}_{e}, \\
D_{S} \leq C l_{\beta}^{-1} d_{S} .
\end{gathered}
$$

Since $\mathscr{S}$ was designed to be adequate for at least $O\left(N^{1 / 2}\right)$ time steps, the number of expected mesh changes is at most $O\left(N^{1 / 2}\right)$. This goal was always achieved in practice.

To avoid rejecting the computed solution $\{\Theta, U\}$ owing to failure of the first test, we always check if the discrete interface $F$ has just reached the boundary of the refined region $\mathscr{R}$, called RED zONE, which in turn alerts that an imminent remeshing must be done; see Figures 9.1 and 9.2. On the other hand, to prevent the program from performing a useless time step owing to failure of either (4.6) or (4.7), these tests can be carried out with more stringent constants. In that case, their failure will only warn that $\mathscr{S}$ cannot be kept any longer. This trick actually succeeds because discrete derivatives may exhibit large oscillations solely near the discrete interface $F$, and thus within $\mathscr{R}$ where the current (local) meshsize is already $O(\tau)$. Hence, remeshing is mostly dictated by the free boundary location, as observed in practice.

In the subsequent analysis of $\S \S 6,7, \mathrm{~S} 4$, we will assume the following structural property which, in view of $\S 3$, is partially justified by Lemma 6.5 and numerical evidence:

$$
\sum_{e \in \mathscr{E}} h_{e}^{2} D_{e} \leq C
$$


The definition of the sets $\mathscr{B}$ and $\widehat{\mathscr{R}}$ might be contradictory unless $\mathscr{B} \subset \mathscr{R}$, that is $h_{S}=O(\tau)$ for all $S \in \mathscr{S}_{B}$. Discrete derivatives $D_{e}$ and $d_{S}$ are typically well-behaved outside $\mathscr{R}$, because this is a parabolic region where $D_{e}, d_{S} \leq$ $C$ at the previous mesh change. Consequently, we do not expect any rapid variation of either $D_{e}$ or $d_{S}$ on $\Omega \backslash \mathscr{R}$, which means $\mathscr{B} \subset \mathscr{R}$ and also $\mathscr{B} \subset$ $\widehat{\mathscr{R}}$. The set $\mathscr{B}$, though, was always empty in our numerical experiments. Note that $\mathscr{F} \subset \widehat{\mathscr{R}}$ as well.

For a well-behaved interface, (4.5) coincides asymptotically with the usual hyperbolic relation $h=O(\tau)$. This was proposed here as a means to balance interpolation errors and attenuate the smearing effect, rather than for stability purposes. Stability is always built into the scheme regardless of the number of mesh changes, as shown in $\S 6$. Our algorithm is still a fixed domain method, even though we predict the region to be invaded by the discrete interface. Indeed, we do not use predicted interfaces to solve uncoupled (nonlinear) heat equations, as customary for front-tracking methods, but rather as a refinement indicator. The behavior of $D_{e}$ and $d_{S}$ depend certainly upon regularity of the underlying problem. We may think of these quantities as being bounded uniformly in $\tau$ away from $F$, where $\theta$ is expected to be smooth; see (3.11). Expressions (4.2) and (4.3), combined with (4.9), then result in $h_{\hat{S}}=O\left(\tau^{1 / 2}\right)$, which is the usual parabolic relation. We finally observe that the assumption $\partial \Omega \in C^{1,1}$ avoids further refinements to alleviate the pollution effect produced by corner singularities; see $\S S 4.4$.

\section{INTERPOLATION ESTIMATES FOR NONCOMPATIBLE MESHES}

Our goal now is to show that the above criteria for mesh selection guarantee a satisfactory error control. The results in $\S 5.1$ are valid in general for regular and noncompatible meshes $\mathscr{S}$ and $\widehat{\mathscr{S}}$ and possess some intrinsic interest. They are next applied to the present setting in $\S \S 5.2,5.3$. We will stick with the notation of $\S 4$.

5.1. The basic estimates. Let us first introduce some further notation and a number of useful geometric properties. Given $W \subset \bar{\Omega}$, set

$$
\mathscr{S}_{W}:=\left\{S \in \mathscr{S}_{0}: S \cap W \neq \varnothing\right\}, \quad \mathscr{E}_{W}:=\left\{e \in \mathscr{E}_{0}: e \cap W \neq \varnothing\right\}, \quad \widetilde{W}:=\bigcup_{S \in \mathscr{S}_{W}} S .
$$

Let $B(x, r)$ indicate the ball of center $x$ and radius $r$. The following facts are simple consequences of the regularity of $\mathscr{S}$ : there exists $0<a<1$ such that for all $S \in \mathscr{S}_{0}$

$$
\begin{gathered}
\rho_{S^{\prime}} \geq a h_{S}, \quad \forall S^{\prime} \in \mathscr{S}_{S}, \\
\operatorname{dist}(x, S) \geq a h_{S}, \quad \forall x \in \widetilde{\Omega}_{0} \backslash \tilde{S}, \\
\operatorname{card} \mathscr{S}_{S}, \quad \text { card } \mathscr{E}_{S}=O(1) .
\end{gathered}
$$

It is then possible to introduce a smooth function $h(x)$ which is locally comparable with the meshsize (see Lemma 5.1 in Supplement $\S \mathrm{S} 1$ ). 
In deriving the interpolation estimates below we shall distinguish between two opposite situations according to the relative size of triangles in both meshes $\mathscr{S}$ and $\widehat{\mathscr{S}}$. It is worth noting that there is no assumption on the relative size or location of new and old triangles at this stage. Set $\widehat{\mathscr{S}}_{0}:=\widehat{\mathscr{S} \backslash}\left(\mathscr{S}_{F} \cup \mathscr{S}_{B}\right)$ and define the derefinement case to be

$$
\text { given } \widehat{S} \in \widehat{\mathscr{S}}_{0}, \quad a h_{S}<h_{\widehat{S}} \quad \text { for all } S \in \mathscr{S}_{\widehat{S}} \text {. }
$$

By contrast, the refinement situation reads as follows

$$
\text { given } \widehat{S} \in \widehat{\mathscr{S}}_{0}, \quad \text { there exists } S \in \mathscr{S}_{\widehat{S}} \quad \text { such that } \quad a h_{S} \geq h_{\widehat{S}} \text {. }
$$

These two cases are obviously mutually exclusive. In addition, for all $\widehat{S}$ 's satisfying (5.5) we have

$$
\widehat{S} \subset \widetilde{S} \quad \text { for some } \quad S \in \mathscr{S}_{\widehat{S}}, \quad \text { card } \mathscr{E}_{\widehat{S}} \text {, card } \mathscr{S}_{\widehat{S}}=O(1),
$$

as results from (5.1), (5.2) and (5.3). Let $\widehat{\mathscr{S}_{1}}$ (resp. $\widehat{\mathscr{S}_{2}}$ ) indicate the set of all $\widehat{S}$ 's satisfying (5.4) (resp. (5.5)). Let $V$ be a generic piecewise linear function defined on $\mathscr{S}$. Let $\zeta: \mathbf{R} \rightarrow \mathbf{R}$ be so that $\zeta^{\prime} \in W^{1, \infty}(\mathbf{R})$. Let $L_{\zeta}$ and $L_{\zeta^{\prime}}$ denote the Lipschitz constants of $\zeta$ and $\zeta^{\prime}$, respectively. Let $\delta_{S}, d_{S}, \delta_{e}$ and $D_{e}$ indicate, for the moment, derivatives extracted from $V$. We now state, and then prove in Supplement $\S S 1$, two crucial estimates, their difference lying essentially in their derivation and further application. The first one refers to the derefinement case and can be viewed as a discrete analogue of that in [2, p. 115]. It roughly asserts that regularity (of $\mathscr{S}$ ) is the sole property that matters for a "discrete" interpolation estimate to hold. The second and more elementary estimate refers to the refinement case (5.5).

Lemma 5.2. Let $\widehat{S} \in \widehat{\mathscr{S}_{1}}$. Then

$$
\begin{aligned}
& \|\Pi \zeta(V)-\hat{\hat{\Pi}} \zeta(V)\|_{L^{p}(\widehat{S})}+h_{\widehat{S}}\|\nabla[\Pi \zeta(V)-\hat{\Pi} \zeta(V)]\|_{L^{p}(\widehat{S})} \\
& \quad \leq h_{\widehat{S}}^{2} \begin{cases}L_{\zeta}\left(\sum_{e \in \mathscr{E}_{\widehat{S}}} h_{e}^{2} D_{e}^{p}\right)^{1 / p}+L_{\zeta^{\prime}}\left(\sum_{S \in \mathcal{S R}} h_{S}^{2} d_{S}^{2 p}\right)^{1 / p}, & 1 \leq p<\infty, \\
L_{\zeta} \max _{e \in \mathbb{S}_{\widehat{S}}} D_{e}+L_{\zeta^{\prime}} \max _{S \in S \mathscr{S}} d_{S}^{2}, & p=\infty .\end{cases}
\end{aligned}
$$


Lemma 5.3. Let $\widehat{S} \in \widehat{\mathscr{S}}_{2}$. Then

$$
\begin{aligned}
& \|\Pi \zeta(V)-\widehat{\Pi} \zeta(V)\|_{L^{p}(\widehat{S})} \\
& \leq \begin{cases}C L_{\zeta} h_{\widehat{S}}^{1+2 / p}\left(\sum_{e \in \mathscr{E}_{\widehat{S}}} \delta_{e}^{p}\right)^{1 / p}+C L_{\zeta^{\prime}} h_{\widehat{S}}^{2 / p}\left(\sum_{S \in \mathscr{S}_{\widehat{S}}} \delta_{S}^{2 p}\right)^{1 / p}, & 1 \leq p<\infty, \\
C L_{\zeta} h_{\widehat{S}} \max _{e \in \mathscr{E}_{S}} \delta_{e}+C L_{\zeta^{\prime}} \max _{S \in \mathscr{S}_{\widehat{S}}} \delta_{S}^{2}, & p=\infty,\end{cases}
\end{aligned}
$$

and

$\|\nabla[\Pi \zeta(V)-\widehat{\Pi} \zeta(V)]\|_{L^{p}(\widehat{S})}$

$$
\leq \begin{cases}C h_{\widehat{S}}^{2 / p}\left[L_{\zeta}\left(\sum_{e \in \mathscr{E}_{\widehat{S}}} \delta_{e}^{p}\right)^{1 / p}+L_{\zeta^{\prime}}\left(\sum_{S \in \mathscr{S}_{\widehat{S}}} h_{S}^{p} d_{S}^{2 p}\right)^{1 / p}\right], & 1 \leq p<\infty, \\ C L_{\zeta} \max _{e \in \mathscr{E}_{S}} \delta_{e}+C L_{\zeta^{\prime}} \max _{S \in \mathscr{S}_{\widehat{S}}}\left(h_{S} d_{S}^{2}\right), & p=\infty .\end{cases}
$$

Remark 5.1. Consider the simplest case $\zeta=$ Identity; thus $L_{\zeta^{\prime}}=0$. We point out that we need control on interpolation errors even for the refinement situation, simply because meshes $\mathscr{S}$ and $\widehat{\mathscr{S}}$ are not compatible. If they were, these errors would simply vanish.

5.2. Error estimates. The first result, whose proof is given in Supplement $\S S 1$, deals with the initial triangulation $\mathscr{S}^{1}$ and the choice (2.12) for the discrete initial enthalpy $U^{0}$.

Lemma 5.4. We have

$$
\left\|u_{0}-U^{0}\right\|_{H^{-1}(\Omega)} \leq C \tau|\log \tau|^{1 / 2} .
$$

We now come to the subtle issue of changing the mesh. Our first task is to apply the two basic interpolation estimates to $U$. To this end, let $V=U$ and $\zeta$ be as in Lemmas 5.2 and 5.3. Note that the choice $\zeta=\beta$ is allowed because, in view of (4.8), it is enough to deal with $\widehat{S} \in \widehat{\mathscr{S}}_{0}$. Recall that derivatives of $U$ in $\Omega_{0}$ can be expressed in terms of quantities extracted from $\Theta$, as stated in (4.10). Hence, let $\delta_{S}, d_{S}, \delta_{e}$, and $D_{e}$ denote from now on derivatives extracted from $\Theta$. The following error estimates illustrate the connection between Lemmas 5.2 and 5.3 and the mesh selection algorithm.

Lemma 5.5. The following sharp pointwise error estimate holds:

$$
\|\Pi \zeta(U)-\hat{\Pi} \zeta(U)\|_{L^{\infty}(\Omega)} \leq C \tau .
$$

Proof. Let first $\widehat{S} \in \widehat{\mathscr{S}_{1}}$. On using (5.7) and (4.10), in conjunction with (4.2), (4.3) and (4.9), we easily obtain

$$
\|\Pi \zeta(U)-\widehat{\Pi} \zeta(U)\|_{L^{\infty}(\widehat{S})} \leq C \max _{e \in \mathscr{Q}_{\widehat{S}}}\left(h_{\widehat{S}}^{2} D_{e}\right)+C \max _{S \in \mathcal{S O}_{\widehat{S}}}\left(h_{\widehat{S}}^{2} d_{S}^{2}\right) \leq C \tau .
$$


Likewise, let us take $\widehat{S} \in \widehat{\mathscr{S}}_{2}$ and make use of (5.8) and (4.10), together with (4.2), (4.3), (4.6) and (4.9), to arrive at

$$
\|\Pi \zeta(U)-\widehat{\Pi} \zeta(U)\|_{L^{\infty}(\widehat{S})} \leq C \max _{e \in \mathscr{E}_{\widehat{S}}}\left(h_{\widehat{S}} h_{e} D_{e}\right)+C \max _{S \in \mathscr{S}_{\widehat{S}}}\left(h_{S}^{2} d_{S}^{2}+h_{\widehat{S}} h_{S} d_{S}^{2}\right) \leq C \tau .
$$

This concludes the proof because $(5.10)$ is obviously sharp.

Remark 5.2. Since meshes $\mathscr{S}$ and $\widehat{\mathscr{S}}$ are not compatible, we cannot expect a pointwise error estimate for $\nabla U$ to hold. To make this claim apparent, we consider, for instance, the refinement case (i.e., $\widehat{S} \in \widehat{\mathscr{S}}_{2}$ ) and suppose that $\zeta:=$ Identity (i.e., $L_{\zeta^{\prime}}=0$ ). We can write (5.9) as follows:

$$
\|\nabla(U-\widehat{U})\|_{L^{\infty}(\widehat{S})} \leq C \max _{e \in \mathscr{E}_{\hat{S}}} h_{e} D_{e}
$$

from which we conclude that $\|\nabla(U-\widehat{U})\|_{L^{\infty}(\widehat{S})}=O(1)$ provided $D_{e}=O\left(h_{e}^{-1}\right)$, as is expected to happen near the interface $F$. In any event, setting $\tilde{h}_{\widehat{S}}:=$ $\min _{e \in \mathscr{E}_{\hat{S}}} \hat{h}_{e}$, we can rewrite (5.9) in the form

$$
\tilde{h}_{\widehat{S}}\|\nabla(U-\widehat{U})\|_{L^{\infty}(\widehat{S})} \leq C \tilde{h}_{\widehat{S}} \max _{e \in \mathscr{E}_{\widehat{S}}} \delta_{e} \leq C \max _{e \in \mathscr{E}_{\hat{S}}}\left(\hat{h}_{e} h_{e} D_{e}\right) \leq C \tau,
$$

as results from (4.2) and (4.6). When $\tilde{h}_{\widehat{S}}=O\left(\tau^{1 / 2}\right)$, as happens away from $F$, we see that

$$
\|\nabla(U-\widehat{U})\|_{L^{\infty}(\widehat{S})} \leq C \tau^{1 / 2} .
$$

It is worth noting that the critical parameter is $\tilde{h}_{\widehat{S}}$ rather than $h_{\widehat{S}}\left(h_{\widehat{S}} \leq \tilde{h}_{\widehat{S}} !\right)$. Similar conclusions hold also for the derefinement case.

In spite of this negative result, we still have an error bound for $\nabla(U-\widehat{U})$ in energy norm. Under the assumptions (4.11) and (3.15), the following lemma yields $\|\nabla[\Pi \zeta(U)-\widehat{\Pi} \zeta(U)]\|_{L^{2}(\Omega)} \leq C \tau^{1 / 2}$.

Lemma 5.6. The following sharp $L^{2}$-error estimate holds:

$$
\|\nabla[\Pi \zeta(U)-\widehat{\Pi} \zeta(U)]\|_{L^{2}(\Omega)}^{2} \leq C \tau\left(L_{\zeta} \sum_{e \in \mathscr{E}_{0}} h_{e}^{2} D_{e}+L_{\zeta^{\prime}} \sum_{S \in \mathscr{S}_{0}} h_{S}^{2} d_{S}^{2}\right) .
$$

Proof. We proceed as in Lemma 5.5 by first examining the derefinement case, namely $\widehat{S} \in \widehat{\mathscr{S}}_{1}$. By virtue of (4.2), (4.3), (4.9) and (4.10), together with (5.7), we easily obtain

$$
\begin{aligned}
\sum_{\widehat{S} \in \widehat{\mathscr{S}}_{1}}\|\nabla[\Pi \zeta(U)-\widehat{\Pi} \zeta(U)]\|_{L^{2}(\widehat{S})}^{2} & \leq C \sum_{\widehat{S} \in \hat{\mathscr{P}}_{1}}\left(\sum_{e \in \mathscr{E}_{\widehat{S}}}\left(h_{\widehat{S}}^{2} D_{e}\right) h_{e}^{2} D_{e}+\sum_{S \in \mathscr{S}_{\widehat{s}}}\left(h_{\widehat{S}}^{2} d_{S}^{2}\right) h_{S}^{2} d_{S}^{2}\right) \\
& \leq C \tau\left(\sum_{e \in \mathscr{E}_{0}} h_{e}^{2} D_{e}+\sum_{S \in \mathscr{S}_{0}} h_{S}^{2} d_{S}^{2}\right) .
\end{aligned}
$$


Note that we could replace double summations by single ones because, as a consequence of (5.4), $\operatorname{card}\left\{\widehat{S} \in \widehat{\mathscr{S}}_{1}: e \cap \widehat{S} \neq \varnothing\right\}=O(1)$ for all $e \in \widehat{\mathscr{E}}_{0}$. On the other hand, for all $\widehat{S} \in \widehat{\mathscr{S}}_{2}$ (refinement case) the above properties, coupled now with (4.6) and (5.9), lead to

$$
\begin{aligned}
\sum_{\widehat{S} \in \hat{\mathscr{S}}_{2}}\|\nabla[\Pi \zeta(U)-\widehat{\Pi} \zeta(U)]\|_{L^{2}(\widehat{S})}^{2} & \leq C \sum_{\widehat{S} \in \widehat{\mathscr{S}}_{2}}\left(\sum_{e \in \mathscr{E}_{S}}\left(h_{\widehat{S}} \delta_{e}\right)^{2}+\sum_{S \in \mathscr{S}_{\widehat{S}}}\left(h_{S}^{2} d_{S}^{2}\right)\left(h_{\widehat{S}}^{2} d_{S}^{2}\right)\right) \\
& \leq C \tau \sum_{\widehat{S} \in \hat{\mathscr{S}}_{2}}\left(\sum_{e \in \mathscr{S}_{S}}\left(h_{\widehat{S}} h_{e} D_{e}\right)+\sum_{S \in \mathscr{S}_{\widehat{S}}} h_{\widehat{S}}^{2} d_{S}^{2}\right) .
\end{aligned}
$$

To proceed further, we need the following elementary inequalities:

$$
\sum_{\widehat{S} \in \widehat{\mathscr{S}}_{e}^{2}} h_{\widehat{S}} \leq C h_{e}, \quad \forall e \in \mathscr{E}_{0}, \quad \sum_{\widehat{S} \in \widehat{\mathscr{S}}_{S}^{2}} h_{\widehat{S}}^{2} \leq C h_{S}^{2}, \quad \forall S \in \mathscr{S}_{0},
$$

where $\widehat{\mathscr{S}}_{e}^{2}:=\left\{\widehat{S} \in \widehat{\mathscr{S}}_{2}: e \cap \widehat{S} \neq \varnothing\right\}$ for all $e \in \mathscr{E}_{0}$ and $\widehat{\mathscr{S}}_{S}^{2}:=\left\{\widehat{S} \in \widehat{\mathscr{S}}_{2}: S \cap \widehat{S} \neq\right.$ $\varnothing\}$ for all $S \in \mathscr{S}_{0}$. Indeed, this yields

$$
\sum_{e \in \mathscr{E}_{0}} h_{e} D_{e} \sum_{\widehat{S} \in \widehat{\mathscr{S}}_{e}^{2}} h_{\widehat{S}} \leq C \sum_{e \in \mathscr{E}_{0}} h_{e}^{2} D_{e}, \quad \sum_{S \in \mathscr{S}_{0}} d_{S}^{2} \sum_{\widehat{S} \in \widehat{\mathscr{S}}_{S}^{2}} h_{\widehat{S}}^{2} \leq C \sum_{S \in \mathscr{S}_{0}} h_{S}^{2} d_{S}^{2},
$$

whence

$$
\sum_{\widehat{S} \in \widehat{\mathscr{S}}_{2}}\|\nabla[\Pi \zeta(U)-\hat{\Pi} \zeta(U)]\|_{L^{2}(\widehat{S})}^{2} \leq C \tau\left(\sum_{e \in \mathscr{E}_{0}} h_{e}^{2} D_{e}+\sum_{S \in \mathscr{S}_{0}} h_{S}^{2} d_{S}^{2}\right) .
$$

It only remains to demonstrate (5.12). The second estimate in (5.12) is obvious in view of (5.5), whereas the first one comes from the following consideration. Let $\widehat{\mathscr{S}}_{e}^{2}:=\left\{\widehat{S}_{k}\right\}_{k=1}^{K}$ be ordered on $e$, and let $x_{k}, x_{k-1}$ be the end points of the segment $e \cap \widehat{S}_{k}$. We would like to replace $h_{k}:=h_{\widehat{S}_{k}}$ by $C\left|x_{k}-x_{k-1}\right|$, but this may not be true for a triangle which is crossed by $e$ near a vertex. We can however argue as follows to overcome the difficulty. The regularity of $\widehat{\mathscr{S}}$ yields card $\mathscr{J}_{k}=O(1)$, where $\mathscr{J}_{k}:=\left\{j: \widehat{S}_{j} \cap B\left(x_{k}, a h_{k}\right) \neq \varnothing\right\}$ for $1 \leq k \leq K$. Hence,

$$
\sum_{k=1}^{K} h_{k} \leq C \sum_{k=1}^{K} \sum_{j \in \mathscr{J}_{k}}\left|x_{j}-x_{j-1}\right| \leq C \sum_{k=1}^{K}\left|x_{k}-x_{k-1}\right| \leq C h_{e} .
$$

Since (5.11) is sharp according to the discrete regularity dealt with, the lemma is thus proved.

Remark 5.3. In view of the pointwise estimate (5.10) and the a priori discrete $L^{1}$-bound (4.11), the energy error estimate (5.11) for $\zeta=$ Identity may be regarded as a 2-D interpolation result, say between $L^{\infty}(\Omega)$ and $W^{2,1}(\Omega)$. 
In $\S 5.3$ we will need two trace estimates for the interpolation error $\Theta-\widehat{\Theta}$ that are stated in Lemmas 5.7 and 5.8 (see Supplement $\S \mathrm{S} 1$ ). They correspond to either the derefinement or the refinement situation.

5.3. A priori estimates. In this subsection we state a priori estimates related to the crucial bound (4.11) as well as (3.15). Their proofs are reported in Supplement $\S S 1$. We begin with (4.11) for the discrete initial temperature $\Theta^{0}:=\Pi^{1}\left[\beta\left(U^{0}\right)\right]=\Pi^{1} \theta_{0}$. The symbol $D_{e}$ stands here for second derivatives extracted from $\Theta^{0}$.

Lemma 5.9. We have

$$
\left\|\nabla \Theta^{0}\right\|_{L^{\infty}(\Omega)}+\sum_{e \in \mathscr{S}^{\prime}} h_{e}^{2} D_{e} \leq C .
$$

We finally state, in Lemmas 5.10 and 5.11, that (4.11) and (3.15) are inherited by $\widehat{\mathscr{S}}$ and $\widehat{\Theta}$. Let $D_{\hat{e}}$ denote the obvious analogue of (4.1) with $\Theta$ replaced by $\widehat{\Theta}$.

Lemma 5.10. The following discrete a priori $W^{2,1}$-bound is valid:

$$
\sum_{\hat{e} \in \hat{\mathscr{E}}} h_{\hat{e}}^{2} D_{\hat{e}} \leq C\left(L_{\beta} \sum_{e \in \mathscr{E}} h_{e}^{2} D_{e}+L_{\beta^{\prime}} \sum_{S \in \mathscr{S}} h_{S}^{2} d_{S}^{2}\right) .
$$

Observe that $\sigma^{2} \sum_{S \in \mathscr{S}} h_{S}^{2} d_{S}^{2} \leq\|\nabla \Theta\|_{L^{2}(\Omega)}^{2} \leq \sum_{S \in \mathscr{S}} h_{S}^{2} d_{S}^{2}$ is valid as well as the obvious analogue with $\Theta$ and $d_{S}$ replaced by $\widehat{\Theta}$ and $d_{\widehat{S}}:=|\nabla \widehat{\Theta}|_{\widehat{S}} \mid$, respectively.

Lemma 5.11. The following discrete a priori $H^{1}$-bound holds:

$$
\|\nabla \widehat{\Theta}\|_{L^{2}(\Omega)}^{2} \leq\left(1+C L_{\beta^{\prime}} \tau\right)\|\nabla \Theta\|_{L^{2}(\Omega)}^{2}+C \tau \sum_{e \in \mathscr{E}_{0}} h_{e}^{2} D_{e} .
$$

Note that the coefficient in front of $\|\nabla \Theta\|_{L^{2}(\Omega)}^{2}$ becomes 1 whenever $\beta$ is piecewise linear, i.e., $L_{\beta^{\prime}}=0$.

\section{Stability}

Our present purpose is to show that the local refinement method (2.13)-(2.15) is stable in various Sobolev norms as soon as the refinement strategy proposed in $\S 4$ is enforced. We start by recalling that $\mathscr{S}^{n}$ is weakly acute, i.e., the sum of the opposite angles with respect of the common side of any pair of faced triangles does not exceed $\pi$. As a consequence, we readily have the following form of the discrete maximum principle: let $\varphi \in \mathbf{V}^{n}$ attain its maximum at the internal node $x_{j}^{n}$ and let $\chi_{j}^{n} \in \mathbf{V}^{n}$ be the corresponding basis function; then

$$
\left\langle\nabla \varphi, \nabla \chi_{j}^{n}\right\rangle \geq 0 \text {. }
$$

This will serve to exploit monotonicity properties of the problem at hand which in turn compensate for the lack of regularity. Note once more the difference between our approach and that for purely parabolic problems [1,5]. The first result, proved in Supplement $\S \mathbf{S} 2$, reads as follows. 
Lemma 6.1. The following a priori maximum norm estimate holds:

$$
\max _{1 \leq n \leq N}\left\|U^{n}\right\|_{L^{\infty}(\Omega)}+\max _{1 \leq n \leq N}\left\|\Theta^{n}\right\|_{L^{\infty}(\Omega)} \leq C .
$$

The following well-known result is also a consequence of (6.1) [3]. We include its proof in Supplement $\S S 2$, just for the sake of completeness.

Lemma 6.2. Let $\alpha \in W^{1, \infty}(\mathbf{R})$ satisfy $\alpha(0)=0$ and $0 \leq \alpha^{\prime}(s) \leq L_{\alpha}<\infty$ for a.e. $s \in \mathbf{R}$. Then

$$
\left\|\nabla \Pi^{n} \alpha(\varphi)\right\|_{L^{2}(\Omega)}^{2} \leq L_{\alpha}\left\langle\nabla \varphi, \nabla \Pi^{n} \alpha(\varphi)\right\rangle, \quad \forall \varphi \in \mathbf{V}^{n} .
$$

In proving the following lemmas, we shall extensively make use of the equivalence of continuous and discrete $L^{2}$-norms for discrete functions, namely

$$
\|\varphi\|_{L^{2}(\Omega)}^{2} \leq\langle\varphi, \varphi\rangle^{n} \leq C\|\varphi\|_{L^{2}(\Omega)}^{2}, \quad \forall \varphi \in \mathbf{V}^{n} .
$$

Our next step is to demonstrate a weak a priori estimate in energy norm. The first term in (6.4) may be thought of as a discrete $H^{1 / 2}\left(0, T ; L^{2}(\Omega)\right)$-norm.

Lemma 6.3. The following a priori estimate holds:

$$
\sum_{n=1}^{N}\left\|U^{n}-\hat{U}^{n-1}\right\|_{L^{2}(\Omega)}^{2}+\sum_{n=1}^{N} \tau\left\|\nabla \Theta^{n}\right\|_{L^{2}(\Omega)}^{2} \leq C .
$$

Proof. Take $\chi=\tau U^{n} \in \mathbf{V}^{n}$ as a test function in (2.15), and next add the resulting expression over $n$ for $1 \leq n \leq m(\leq N)$. We have

$$
\begin{aligned}
\mathrm{I}+\mathrm{II} & :=\sum_{n=1}^{m}\left\langle U^{n}-\hat{U}^{n-1}, U^{n}\right\rangle^{n}+\sum_{n=1}^{m} \tau\left\langle\nabla \boldsymbol{\Theta}^{n}, \nabla U^{n}\right\rangle \\
& =\sum_{n=1}^{m} \tau\left\langle f\left(\hat{\boldsymbol{\Theta}}^{n-1}\right), U^{n}\right\rangle^{n}=: \text { III. }
\end{aligned}
$$

Using (2.16) together with the elementary identity

$$
2 a(a-b)=a^{2}-b^{2}+(a-b)^{2}, \quad \forall a, b \in \mathbf{R},
$$

the first term can be further split as follows $\left(\Pi^{0}:=\Pi^{1}\right)$ :

$$
\begin{aligned}
2 \mathrm{I}= & \sum_{n=1}^{m} \int_{\Omega} \Pi^{n}\left[\left(U^{n}\right)^{2}\right]-\Pi^{n}\left[\left(U^{n-1}\right)^{2}\right]+\Pi^{n}\left[\left(U^{n}-U^{n-1}\right)^{2}\right] \\
= & \sum_{n=1}^{m} \int_{\Omega} \Pi^{n}\left[\left(U^{n}\right)^{2}\right]-\Pi^{n-1}\left[\left(U^{n-1}\right)^{2}\right] \\
& +\sum_{n=2}^{m} \int_{\Omega} \Pi^{n-1}\left[\left(U^{n-1}\right)^{2}\right]-\Pi^{n}\left[\left(U^{n-1}\right)^{2}\right] \\
& +\sum_{n=1}^{m} \int_{\Omega} \Pi^{n}\left[\left(U^{n}-U^{n-1}\right)^{2}\right]=: \mathrm{I}_{1}+\mathrm{I}_{2}+\mathrm{I}_{3} .
\end{aligned}
$$


We now evaluate these three terms separately. We first obtain

$$
\mathrm{I}_{1}=\left\langle U^{m}, U^{m}\right\rangle^{m}-\left\langle U^{0}, U^{0}\right\rangle^{1} \geq\left\|U^{m}\right\|_{L^{2}(\Omega)}^{2}-C\left\|U^{0}\right\|_{L^{2}(\Omega)}^{2},
$$

and

$$
\mathrm{I}_{3}=\sum_{n=1}^{m}\left\langle U^{n}-\widehat{U}^{n-1}, U^{n}-\widehat{U}^{n-1}\right\rangle^{n} \geq \sum_{n=1}^{m}\left\|U^{n}-\widehat{U}^{n-1}\right\|_{L^{2}(\Omega)}^{2} .
$$

The remaining term, which occurs only when the mesh is changed, can be handled by Lemma 5.5 with $\zeta(s)=s^{2}$. Indeed, since $U^{n}$ satisfies $(6.2), \zeta$ can be suitably modified outside the range of $U^{n}$ in such a way that $\zeta^{\prime} \in W^{1, \infty}(\mathbf{R})$. Hence $\mathrm{I}_{2} \geq-C \sum_{n=2}^{m} \tau=-C$. On the other hand, (2.13) in conjunction with (2.1) and Lemma 6.2 yields

$$
\sum_{n=1}^{m} \tau\left\|\nabla \Theta^{n}\right\|_{L^{2}(\Omega)}^{2} \leq L_{\beta} \mathrm{II}
$$

For the remaining term III we make use of (6.2) to arrive at

$$
|\mathrm{III}| \leq \sum_{n=1}^{m} \tau\left(|f(0)| \operatorname{meas}(\Omega)^{1 / 2}+L_{f}\left\|\widehat{\Theta}^{n-1}\right\|_{L^{2}(\Omega)}\right)\left\|U^{n}\right\|_{L^{2}(\Omega)} \leq C,
$$

because $\widehat{\Theta}^{n-1}=\Pi^{n}\left[\beta\left(U^{n-1}\right)\right]$. The proof is thus complete.

We now derive a strong a priori estimate in energy norms. To this end, we need the structural assumption (4.11).

Lemma 6.4. Let (4.11) hold. Then

$$
\sum_{n=1}^{N} \tau^{-1}\left\|\Theta^{n}-\widehat{\Theta}^{n-1}\right\|_{L^{2}(\Omega)}^{2}+\max _{1 \leq n \leq N}\left\|\nabla \Theta^{n}\right\|_{L^{2}(\Omega)} \leq C .
$$

Proof. We argue by induction. Let $1=: n_{1}<n_{2}<\cdots<n_{K} \leq N$ denote the indexes corresponding to the mesh changes; set $n_{K+1}:=N+1$. We want to prove the following inequality for all $m$ between two successive mesh changes, say $n_{k} \leq m<n_{k+1}$ :

$$
\begin{aligned}
A_{m} & :=\left(2 L_{\beta}\right)^{-1} \sum_{n=1}^{m} \tau^{-1}\left\|\Theta^{n}-\widehat{\Theta}^{n-1}\right\|_{L^{2}(\Omega)}^{2}+\max _{0 \leq n \leq m}\left\|\nabla \Theta^{n}\right\|_{L^{2}(\Omega)}^{2} \\
& \leq C_{m}:=\exp \left(C L_{\beta^{\prime}} m \tau\right)\left(C\left(B_{k}+1\right) m \tau+\left\|\nabla \Theta^{0}\right\|_{L^{2}(\Omega)}^{2}\right)
\end{aligned}
$$

where $B_{k}:=\max _{1 \leq i \leq k} \sum_{e \in \mathscr{E}_{0}^{n_{i}-1}} h_{e}^{2} D_{e}^{n_{i}-1}$. Since this estimate is obviously valid for $m=0$, assume, by induction, that it holds for all $m<n_{k}$ as well. 
Take $\chi=\Theta^{n}-\widehat{\Theta}^{n-1} \in \mathbf{V}^{n}$ as a test function in (2.15) and next add the resulting equality from $n=n_{k}$ to $n=m\left(<n_{k+1}\right)$. We end up with

$$
\begin{aligned}
\mathrm{I}+\mathrm{II}:= & \sum_{n=n_{k}}^{m} \tau^{-1}\left\langle U^{n}-\Pi^{n} U^{n-1}, \Pi^{n}\left[\beta\left(U^{n}\right)-\beta\left(U^{n-1}\right)\right]\right\rangle^{n} \\
& +\sum_{n=n_{k}}^{m}\left\langle\nabla \boldsymbol{\Theta}^{n}, \nabla\left(\boldsymbol{\Theta}^{n}-\widehat{\Theta}^{n-1}\right)\right\rangle \\
= & \sum_{n=n_{k}}^{m}\left\langle f\left(\hat{\boldsymbol{\Theta}}^{n-1}\right), \Theta^{n}-\widehat{\Theta}^{n-1}\right\rangle^{n}=: \text { III. }
\end{aligned}
$$

The first term can be easily evaluated as follows:

$$
\begin{aligned}
\mathrm{I} & =\sum_{n=n_{k}}^{m} \tau^{-1} \int_{\Omega} \Pi^{n}\left[\left(U^{n}-U^{n-1}\right)\left(\beta\left(U^{n}\right)-\beta\left(U^{n-1}\right)\right)\right] \\
& \geq L_{\beta}^{-1} \sum_{n=n_{k}}^{m} \tau^{-1} \int_{\Omega} \Pi^{n}\left[\left(\beta\left(U^{n}\right)-\beta\left(U^{n-1}\right)\right)^{2}\right] \\
& \geq L_{\beta}^{-1} \sum_{n=n_{k}}^{m} \tau^{-1}\left\|\Theta^{n}-\hat{\Theta}^{n-1}\right\|_{L^{2}(\Omega)}^{2} .
\end{aligned}
$$

Since $\widehat{\Theta}^{n-1}=\Theta^{n-1}$ for all $n_{k}<n<n_{k+1}$, as a by-product of (6.5), the next term becomes

$$
2 \mathrm{II}=\left\|\nabla \boldsymbol{\Theta}^{m}\right\|_{L^{2}(\Omega)}^{2}-\left\|\nabla \widehat{\Theta}^{n_{k}-1}\right\|_{L^{2}(\Omega)}^{2}+\sum_{n=n_{k}}^{m}\left\|\nabla\left(\boldsymbol{\Theta}^{n}-\widehat{\Theta}^{n-1}\right)\right\|_{L^{2}(\Omega)}^{2} .
$$

By virtue of the induction assumption we can use (5.14) to arrive at

$$
\begin{aligned}
& \left\|\nabla \widehat{\Theta}^{n_{k}-1}\right\|_{L^{2}(\Omega)}^{2} \leq\left(1+C L_{\beta^{\prime}} \tau\right)\left\|\nabla \Theta^{n_{k}-1}\right\|_{L^{2}(\Omega)}^{2}+C \tau \sum_{e \in \mathscr{E}_{0}^{n_{k}-1}} h_{e}^{2} D_{e}^{n_{k}-1} \\
& \leq\left(1+C L_{\beta^{\prime}} \tau\right) \exp \left(C L_{\beta^{\prime}}\left(n_{k}-1\right) \tau\right)\left(C\left(B_{k-1}+1\right)\left(n_{k}-1\right) \tau+\left\|\nabla \Theta^{0}\right\|_{L^{2}(\Omega)}^{2}\right) \\
& \quad+C B_{k} \tau-\left(\left(2 L_{\beta}\right)^{-1}+C \tau\right) \sum_{n=1}^{n_{k}-1} \tau^{-1}\left\|\Theta^{n}-\widehat{\Theta}^{n-1}\right\|_{L^{2}(\Omega)}^{2},
\end{aligned}
$$

whence

$$
\begin{aligned}
& \left\|\nabla \widehat{\boldsymbol{\Theta}}^{n_{k}-1}\right\|_{L^{2}(\Omega)}^{2}+\left(2 L_{\beta}\right)^{-1} \sum_{n=1}^{n_{k}-1} \tau^{-1}\left\|\Theta^{n}-\widehat{\Theta}^{n-1}\right\|_{L^{2}(\Omega)}^{2} \\
& \quad \leq \exp \left(C L_{\beta^{\prime}} n_{k} \tau\right)\left(C\left(B_{k-1}+1\right)\left(n_{k}-1\right) \tau+\left\|\nabla \Theta^{0}\right\|_{L^{2}(\Omega)}^{2}\right)+C B_{k} \tau,
\end{aligned}
$$

because $1+C L_{\beta} \tau \leq \exp \left(C L_{\beta} \tau\right)$. It only remains to examine term III, for 
which we have

$$
\begin{aligned}
|\mathrm{III}| & \leq \sum_{n=n_{k}}^{m}\left(|f(0)| \operatorname{meas}(\Omega)^{1 / 2}+L_{f}\left\|\widehat{\Theta}^{n-1}\right\|_{L^{2}(\Omega)}\right)\left\|\Theta^{n}-\widehat{\Theta}^{n-1}\right\|_{L^{2}(\Omega)} \\
& \leq C\left(m-n_{k}+1\right) \tau+\left(2 L_{\beta}\right)^{-1} \sum_{n=n_{k}}^{m} \tau^{-1}\left\|\Theta^{n}-\widehat{\Theta}^{n-1}\right\|_{L^{2}(\Omega)}^{2}
\end{aligned}
$$

because (6.2) yields $\left\|\widehat{\Theta}^{n-1}\right\|_{L^{2}(\Omega)} \leq C\left\|\Theta^{n-1}\right\|_{L^{\infty}(\Omega)} \leq C$. Collecting all partial results, we get the following estimate, for any $n_{k} \leq m<n_{k+1}$,

$$
\begin{aligned}
A_{m} \leq & \exp \left(C L_{\beta^{\prime}} n_{k} \tau\right)\left(C\left(B_{k-1}+1\right)\left(n_{k}-1\right) \tau+\left\|\nabla \Theta^{0}\right\|_{L^{2}(\Omega)}^{2}\right) \\
& +C B_{k} \tau+C\left(m-n_{k}+1\right) \tau \\
\leq & \exp \left(C L_{\beta^{\prime}} m \tau\right)\left(C\left(B_{k}+1\right) m \tau+\left\|\nabla \Theta^{0}\right\|_{L^{2}(\Omega)}^{2}\right)=C_{m} .
\end{aligned}
$$

The induction argument is thus complete. Finally, the desired estimate follows from Lemma 5.9 and assumption (4.11).

Remark 6.1. As a by-product of the above argument, combined with (2.1), (2.13) and the fact that $F^{n-1}, F^{n} \subset \mathscr{R}^{n}$ for all $1 \leq n \leq N$, we deduce

$$
\begin{aligned}
C & \geq \sum_{n=1}^{N} \tau^{-1} \int_{\Omega} \Pi^{n}\left[\left(U^{n}-U^{n-1}\right)^{2} \int_{0}^{1} \beta^{\prime}\left(s U^{n}+(1-s) U^{n-1}\right) d s\right] \\
& \geq l_{\beta} \sum_{n=1}^{N} \tau^{-1} \int_{\Omega \backslash \mathscr{R}^{n}} \Pi^{n}\left[\left(U^{n}-U^{n-1}\right)^{2}\right],
\end{aligned}
$$

whence

$$
\sum_{n=1}^{N} \tau^{-1}\left\|U^{n}-\widehat{U}^{n-1}\right\|_{L^{2}\left(\Omega \backslash \mathscr{R}^{n}\right)}^{2} \leq C .
$$

Our final estimate is a discrete analogue of (2.9): $u_{t} \in L^{\infty}(0, T ; M(\Omega))$.

Lemma 6.5. Let (4.11) hold. Then

$$
\max _{1 \leq n \leq N}\left\|U^{n}-\widehat{U}^{n-1}\right\|_{L^{1}(\Omega)} \leq C \tau \text {. }
$$

Proof. It is enough to prove (6.8) for all steps between two successive mesh changes, say $1 \leq n_{k} \leq m<n_{k+1}$. Moreover, we can assume without loss of generality that $\beta$ is strictly increasing because the asymptotic constant in (6.8) is independent of the lower bound of $\beta^{\prime}$. As a result, $\beta^{-1}$ is well defined and monotone increasing. Subtract now two consecutive discrete equations (2.15) for $n_{k} \leq n<n_{k+1}$ to arrive at

$$
\left\langle\partial U^{n}-\partial U^{n-1}, \chi\right\rangle^{n_{k}}+\tau\left\langle\nabla \partial \Theta^{n}, \nabla \chi\right\rangle=\tau\left\langle\partial f\left(\boldsymbol{\Theta}^{n-1}\right), \chi\right\rangle^{n_{k}},
$$


where $\partial z^{n}:=\tau^{-1}\left(z^{n}-z^{n-1}\right)$ and $\Theta^{n_{k}-2}:=\Theta^{n_{k}-1}$. For notational convenience, we have used $U^{n_{k}-1}$ to denote $\hat{U}^{n_{k}-1}=\Pi^{n_{k}}\left(U^{n_{k}-1}\right) \in \mathbf{V}^{n_{k}}, \Theta^{n_{k}-1}$ to designate $\hat{\boldsymbol{\Theta}}^{n_{k}-1}=\Pi^{n_{k}} \beta\left(U^{n_{k}-1}\right) \in \mathbf{V}^{n_{k}}$ and, finally, $U^{n_{k}-2} \in \mathbf{V}^{n_{k}}$ to indicate the solution of the following auxiliary problem:

$$
\tau^{-1}\left\langle U^{n_{k}-1}-U^{n_{k}-2}, \chi\right\rangle^{n_{k}}+\left\langle\nabla \Theta^{n_{k}-1}, \nabla \chi\right\rangle=\left\langle f\left(\Theta^{n_{k}-1}\right), \chi\right\rangle^{n_{k}}, \quad \forall \chi \in \mathbf{V}^{n_{k}} .
$$

By virtue of (2.15), (2.18) and (6.2), we readily have for all $\chi \in \mathbf{V}^{n_{k}},\|\chi\|_{L^{\infty}(\Omega)}$ $\leq 1$,

$$
\begin{aligned}
\left|\left\langle\partial U^{n_{k}-1}, \chi\right\rangle^{n_{k}}\right| & \leq\left|\left\langle f\left(\Theta^{n_{k}-1}\right), \chi\right\rangle^{n_{k}}\right|+\sum_{e \in \mathscr{E}^{n_{k}}} \mid\left\langle\left[\mathbb{[} \nabla \boldsymbol{\Theta}^{n_{k}-1} \mathbb{1}_{e} \cdot \nu_{e}, \chi\right\rangle_{e}\right| \\
& \leq C\left(1+\sum_{e \in \mathscr{E}^{n_{k}}} h_{e}^{2} D_{e}^{n_{k}-1}\right) .
\end{aligned}
$$

Let $\psi_{\varepsilon} \in C^{\infty}(\mathbf{R})$ be a monotone approximation of the function sgn such that $\psi_{\varepsilon}(0)=0,\left|\psi_{\varepsilon}(s)\right| \leq 1$ and $\psi_{\varepsilon}(s) \rightarrow \operatorname{sgn}(s)$ as $\varepsilon \downarrow 0$ for all $s \in \mathbf{R} \backslash\{0\}$. Set $\chi:=\Pi^{n_{k}}\left[\psi_{\varepsilon}\left(\partial U^{n_{k}-1}\right)\right]$ in the above inequality and let $\varepsilon \downarrow 0$. Since $\boldsymbol{\Theta}^{n_{k}-1}$ is either the initial discrete temperature or that corresponding to a mesh change, it satisfies either Lemma 5.9 or Lemma 5.10. We infer that

$$
\int_{\Omega} \Pi^{n_{k}}\left|\partial U^{n_{k}-1}\right| \leq C
$$

because of (4.11) and (3.15). To proceed further, take $\chi:=\Pi^{n_{k}}\left[\psi_{\varepsilon}\left(\partial \Theta^{n}\right)\right] \in \mathbf{V}^{n_{k}}$ as a test function in (6.9) and next add the resulting expressions from $n_{k}$ to $m<n_{k+1}$. We obtain

$$
\begin{aligned}
\mathrm{I}+\mathrm{II}:= & \sum_{n=n_{k}}^{m}\left\langle\partial\left(U^{n}-U^{n-1}\right), \Pi^{n_{k}}\left[\psi_{\varepsilon}\left(\partial \Theta^{n}\right)\right]\right\rangle^{n_{k}} \\
& +\sum_{n=n_{k}}^{m} \tau\left\langle\nabla \partial \Theta^{n}, \nabla \Pi^{n_{k}}\left[\psi_{\varepsilon}\left(\partial \Theta^{n}\right)\right]\right\rangle \\
= & \sum_{n=n_{k}}^{m} \tau\left\langle\partial f\left(\Theta^{n-1}\right), \Pi^{n_{k}}\left[\psi_{\varepsilon}\left(\partial \Theta^{n}\right)\right]\right\rangle^{n_{k}}=: \text { III, }
\end{aligned}
$$

which is now examined in detail. By virtue of $(6.3)$ we have II $\geq 0$. For III we make use of (6.6) to arrive at

$$
|\mathrm{III}| \leq C \sum_{n=n_{k}}^{m-1} \tau L_{f}\left\|\partial \Theta^{n}\right\|_{L^{2}(\Omega)} \leq C .
$$

For the remaining term I we reason as follows. We first observe that

$$
0 \leq \partial U^{n-1}\left(x_{j}^{n_{k}}\right) \psi_{\varepsilon}\left(\partial \Theta^{n}\left(x_{j}^{n_{k}}\right)\right) \leq\left|\partial U^{n-1}\left(x_{j}^{n_{k}}\right)\right|,
$$

for all nodes $x_{j}^{n_{k}}$ of $\mathscr{S}^{n_{k}}$. Secondly, we take the limit as $\varepsilon \downarrow 0$ to obtain

$$
\begin{aligned}
\partial U^{n}\left(x_{j}^{n_{k}}\right) \psi_{\varepsilon}\left(\partial \Theta^{n}\left(x_{j}^{n_{k}}\right)\right) & \rightarrow \partial U^{n}\left(x_{j}^{n_{k}}\right) \operatorname{sgn}\left(\partial \Theta^{n}\left(x_{j}^{n_{k}}\right)\right) \\
& =\partial U^{n}\left(x_{j}^{n_{k}}\right) \operatorname{sgn}\left(\partial U^{n}\left(x_{j}^{n_{k}}\right)\right)=\left|\partial U^{n}\left(x_{j}^{n_{k}}\right)\right|,
\end{aligned}
$$


for all $x_{j}^{n_{k}}$ such that $\partial U^{n}\left(x_{j}^{n_{k}}\right) \neq 0$ or, equivalently, $\partial \Theta^{n}\left(x_{j}^{n_{k}}\right) \neq 0$. Note that it is precisely here where we need $\beta$ to be strictly increasing. Therefore, term I becomes

$$
\sum_{n=n_{k}}^{m} \int_{\Omega} \Pi^{n_{k}}\left|\partial U^{n}\right|-\int_{\Omega} \Pi^{n_{k}}\left|\partial U^{n-1}\right|=\int_{\Omega} \Pi^{n_{k}}\left|\partial U^{m}\right|-\int_{\Omega} \Pi^{n_{k}}\left|\partial U^{n_{k}-1}\right| .
$$

Collecting the previous estimates and making use of (6.10), combined with the fact that $\|\chi\|_{L^{1}(\Omega)} \leq \int_{\Omega} \Pi^{n_{k}}|\chi|$ for all $\chi \in \mathbf{V}^{n_{k}}$, the assertion easily follows with an asymptotic constant independent of the lower bound of $\beta^{\prime}$.

\section{ENERGY ERROR ESTIMATES}

In this section we derive error estimates in the natural energy spaces for our Adaptive Method. We also state a quasi-optimal error estimate in the maximum norm for an auxiliary elliptic problem which may have some independent interest. Its proof is reported in Supplement $\S \mathbf{S} 3$. We stress once again that technical arguments to handle the discrepancy between continuous and discrete domains were introduced in full detail in [17] and are thus omitted here by simply assuming $\Omega^{n}=\bar{\Omega}$.

We start by recalling a well-known interpolation estimate for the quadrature formula (2.16), namely, for all $S \in \mathscr{S}^{n}$ we have

$$
\int_{S}\left|\chi \varphi-\Pi^{n}(\chi \varphi)\right| d x \leq C h_{S}\|\chi\|_{L^{2}(S)}\|\nabla \varphi\|_{L^{2}(S)}, \quad \forall \chi, \varphi \in \mathbf{V}^{n} .
$$

Let $G: H^{-1}(\Omega) \rightarrow H_{0}^{1}(\Omega)$ designate the Green's operator, that is,

$$
\langle\nabla G \psi, \nabla \varphi\rangle=\langle\psi, \varphi\rangle, \quad \forall \varphi \in H_{0}^{1}(\Omega) .
$$

Since $\Omega$ is smooth, the operator $G$ is regular [2, p. 138]:

$$
\|G \psi\|_{H^{2}(\Omega)} \leq C\|\psi\|_{L^{2}(\Omega)}, \quad \forall \psi \in L^{2}(\Omega) .
$$

In addition, the norm in $H^{-1}(\Omega)$ can be represented in terms of $G$ as follows:

$$
\|\psi\|_{H^{-1}(\Omega)}=\|\nabla G \psi\|_{L^{2}(\Omega)}=\langle\psi, G \psi\rangle^{1 / 2}, \quad \forall \psi \in H^{-1}(\Omega) .
$$

The discrete Green's operator $G^{n}: H^{-1}(\Omega) \rightarrow \mathbf{V}^{n}$ is defined by

$$
\left\langle\nabla G^{n} \psi, \nabla \chi\right\rangle=\langle\psi, \chi\rangle, \quad \forall \chi \in \mathbf{V}^{n},
$$

and satisfies the following error estimates. Set $h_{n}:=\max _{S \in \mathcal{S}^{n}} h_{S}, \rho_{n}:=$ $\min _{S \in \mathcal{S}^{n}} h_{S}$ and recall that $\lambda \tau \leq \rho_{n}<h_{n} \leq \Lambda \tau^{1 / 2}$, as results from (4.9). We have first

$$
\left\|\left(G-G^{n}\right) \psi\right\|_{L^{2}(\Omega)} \leq C h_{n}^{2}\|\psi\|_{L^{2}(\Omega)}, \quad \forall \psi \in L^{2}(\Omega),
$$

under the sole assumption of regularity of both $\mathscr{S}^{n}$ and $G$ [2]. If, in addition, $h_{n} \leq C \rho_{n}^{\gamma}$ with $0<\gamma \leq 1$, we will prove in $\S \mathrm{S} 3.2$ the following quasi-optimal pointwise estimate; in the present case $\gamma=1 / 2$. 
Lemma 7.1. There holds $\left\|\left(G-G^{n}\right) \psi\right\|_{L^{\infty}(\Omega)} \leq C h_{n}^{2}\left|\log h_{n}\right|^{7}\|\psi\|_{L^{\infty}(\Omega)}$, for all $\psi \in L^{\infty}(\Omega)$.

Its proof is based on having suitable local energy estimates as in [21, 22, 23]. However, the novelty here is that $\mathscr{S}^{n}$ is not quasi-uniform nor does it have any a priori structure as in [22]. Such a new difficulty is responsible for the extra power of the logarithm.

We now introduce the error equations. Integration of (2.8) on the interval $I^{n}:=\left(t^{n-1}, t^{n}\right]$ results in

$$
\begin{aligned}
\left\langle u^{n}-u^{n-1}, \varphi\right\rangle & +\left\langle\int_{I^{n}} \nabla \theta(t) d t, \nabla \varphi\right\rangle \\
= & \left\langle\int_{I^{n}} f(\theta(t)) d t, \varphi\right\rangle, \forall \varphi \in H_{0}^{1}(\Omega), 1 \leq n \leq N,
\end{aligned}
$$

where $t^{n}:=n \tau$ and $u^{n}:=u\left(t^{n}\right)$. At the same time, (2.15) can be written equivalently as

$$
\begin{aligned}
\left\langle U^{n}-\right. & \left.U^{n-1}, \varphi\right\rangle+\tau\left\langle\nabla \Theta^{n}, \nabla \chi\right\rangle \\
= & \left\langle U^{n}-\widehat{U}^{n-1}, \chi\right\rangle-\left\langle U^{n}-\widehat{U}^{n-1}, \chi\right\rangle^{n} \\
& +\left\langle U^{n}-\widehat{U}^{n-1}, \varphi-\chi\right\rangle+\left\langle\widehat{U}^{n-1}-U^{n-1}, \varphi\right\rangle \\
& +\tau\left\langle f\left(\widehat{\Theta}^{n-1}\right), \chi\right\rangle^{n}, \quad \forall \varphi \in H_{0}^{1}(\Omega), \chi \in \mathbf{V}^{n}, \quad 1 \leq n \leq N .
\end{aligned}
$$

Subtraction of (7.8) from (7.7) yields the error equation

$$
\begin{aligned}
\left\langle e_{u}^{n}-\right. & \left.e_{u}^{n-1}, \varphi\right\rangle+\left\langle\int_{I^{n}} \nabla e_{\theta}(t) d t, \nabla \varphi\right\rangle+\tau\left\langle\nabla \Theta^{n}, \nabla(\varphi-\chi)\right\rangle \\
= & \left\langle U^{n}-\widehat{U}^{n-1}, \chi\right\rangle^{n}-\left\langle U^{n}-\widehat{U}^{n-1}, \chi\right\rangle+\left\langle\widehat{U}^{n-1}-U^{n}, \varphi-\chi\right\rangle \\
& \quad+\left\langle U^{n-1}-\widehat{U}^{n-1}, \varphi\right\rangle+\left\langle\int_{I^{n}} f(\theta(t)) d t, \varphi\right\rangle-\tau\left\langle f\left(\widehat{\Theta}^{n-1}\right), \chi\right\rangle^{n},
\end{aligned}
$$

for all $\varphi \in H_{0}^{1}(\Omega), \chi \in \mathbf{V}^{n}$, where we have set, for any $1 \leq n \leq N$,

$$
e_{u}(t):=u(t)-U^{n}, \quad e_{\theta}(t):=\theta(t)-\Theta^{n}, \quad \forall t \in I^{n}, \quad \text { and } \quad e_{u}^{n}:=e_{u}\left(t^{n}\right) .
$$

Theorem 7.1. Let (4.11) hold and the number of mesh changes be bounded above by $O\left(\tau^{-1 / 2}\right)$. Then

$$
\left\|e_{u}\right\|_{L^{\infty}\left(0, T ; H^{-1}(\Omega)\right)}+\left\|e_{\theta}\right\|_{L^{2}\left(0, T ; L^{2}(\Omega)\right)} \leq C \tau^{1 / 2}|\log \tau|^{7 / 2} .
$$

For the practical range of time steps $\tau$, the above rate is essentially $O\left(\tau^{1 / 2}\right)$. The restriction on the number of mesh changes accounts for the accumulation of interpolation errors $U^{n-1}-\widehat{U}^{n-1}$ which, by Lemma 5.5, are $O(\tau)$. The mesh selection algorithm of $\S 4.2$ is so designed as to make such a restriction and (4.11) acceptable constraints. The key issue in Theorem 7.1 is the underlying set of graded meshes $\left\{\mathscr{S}^{n}\right\}_{n=1}^{N}$ for which it holds and constitutes the first rigorous result. In fact, similar rates of convergence have been obtained for 
quasi-uniform meshes $[4,8,17,24]$. The improvement upon those results is thus to be expressed in terms of spatial degrees of freedom, as explained in $\S 8.2$.

Proof of Theorem 7.1. Take first $\varphi:=G e_{u}^{n} \in H_{0}^{1}(\Omega)$ and $\chi:=G^{n} e_{u}^{n} \in \mathbf{V}^{n}$ in (7.9), next add over $n$ from 1 to $m(\leq N)$ and use (7.2) and (7.5) to arrive at

$$
\begin{aligned}
\sum_{n=1}^{m}\left\langle e_{u}^{n}-e_{u}^{n-1}, G e_{u}^{n}\right\rangle+\sum_{n=1}^{m}\left\langle\int_{I^{n}} e_{\theta}(t) d t, e_{u}^{n}\right\rangle=: \mathrm{I}+\mathrm{II} \\
=\sum_{n=1}^{m}\left[\left\langle U^{n}-\widehat{U}^{n-1}, G^{n} e_{u}^{n}\right\rangle^{n}-\left\langle U^{n}-\widehat{U}^{n-1}, G^{n} e_{u}^{n}\right\rangle\right] \\
\quad+\sum_{n=1}^{m}\left\langle\widehat{U}^{n-1}-U^{n},\left(G-G^{n}\right) e_{u}^{n}\right\rangle+\sum_{n=1}^{m}\left\langle U^{n-1}-\widehat{U}^{n-1}, G e_{u}^{n}\right\rangle \\
\quad+\sum_{n=1}^{m}\left(\left\langle\int_{I^{n}} f(\theta(t)) d t, G e_{u}^{n}\right\rangle-\tau\left\langle f\left(\widehat{\Theta}^{n-1}, G^{n} e_{u}^{n}\right\rangle^{n}\right)\right. \\
=: \text { III }+\cdots+\text { VI. }
\end{aligned}
$$

The rest of the proof consists simply of evaluating these six terms separately. In order to simplify notation, set

$$
A_{m}:=\max _{1 \leq n \leq m}\left\|e_{u}^{n}\right\|_{H^{-1}(\Omega)}^{2} .
$$

By virtue of Lemma 5.4, (6.5) and (7.4), the first term yields

$$
2 \mathrm{I}=\left\|e_{u}^{m}\right\|_{H^{-1}(\Omega)}^{2}-\left\|u_{0}-U^{0}\right\|_{H^{-1}(\Omega)}^{2}+\sum_{n=1}^{m}\left\|e_{u}^{n}-e_{u}^{n-1}\right\|_{H^{-1}(\Omega)}^{2} \geq\left\|e_{u}^{m}\right\|_{H^{-1}(\Omega)}^{2}-C \tau \text {. }
$$

In view of the constitutive relations (2.6) and (2.13), term II can be further split as follows:

$$
\begin{aligned}
\mathrm{II}_{1}+\mathrm{II}_{2}+\mathrm{II}_{3}:= & \sum_{n=1}^{m} \int_{I^{n}}\left\langle e_{\theta}(t), u\left(t^{n}\right)-u(t)\right\rangle d t \\
& +\sum_{n=1}^{m} \int_{I^{n}}\left\langle\beta(u(t))-\beta\left(U^{n}\right), u(t)-U^{n}\right\rangle d t \\
& +\sum_{n=1}^{m} \int_{I^{n}}\left\langle\beta\left(U^{n}\right)-\Pi^{n} \beta\left(U^{n}\right), e_{u}(t)\right\rangle d t .
\end{aligned}
$$

We first make use of (2.5) and (6.4) to evaluate term $\mathrm{II}_{1}$ as follows:

$$
\left|\mathrm{II}_{1}\right| \leq \sum_{n=1}^{m} \int_{I^{n}}\left\|\nabla e_{\theta}(t)\right\|_{L^{2}(\Omega)}\left\|\int_{t}^{t^{n}} u_{t}(s) d s\right\|_{H^{-1}(\Omega)} d t \leq C \tau .
$$

We next recall an elementary interpolation estimate. Let $\alpha: \mathbf{R} \rightarrow \mathbf{R}$ be a continuous and nondecreasing function; then

$$
\begin{aligned}
& \left\|\alpha(\varphi)-\Pi^{n} \alpha(\varphi)\right\|_{L^{p}(S)} \\
& \quad \leq C h_{S}\left\|\nabla \Pi^{n} \alpha(\varphi)\right\|_{L^{p}(S)}, \quad \forall S \in \mathscr{S}^{n}, \varphi \in \mathbf{V}^{n}, 1 \leq p \leq \infty .
\end{aligned}
$$


Applying this inequality with $\alpha=\beta$ and $p=2$, in conjunction with (6.4) and the constraint $h_{S} \leq \Lambda \tau^{1 / 2}$ for all $S \in \mathscr{S}^{n}$, we obtain

$$
\begin{aligned}
\mathrm{II}_{2} & \geq\left(2 L_{\beta}\right)^{-1} \sum_{n=1}^{m} \int_{I^{n}}\left\|e_{\theta}(t)\right\|_{L^{2}(\Omega)}^{2} d t-L_{\beta}^{-1} \tau \sum_{n=1}^{m}\left\|\beta\left(U^{n}\right)-\Pi^{n} \beta\left(U^{n}\right)\right\|_{L^{2}(\Omega)}^{2} \\
& \geq\left(2 L_{\beta}\right)^{-1}\left\|e_{\theta}\right\|_{L^{2}\left(0, t^{m} ; L^{2}(\Omega)\right)}^{2}-C \tau .
\end{aligned}
$$

The analysis of term $\mathrm{II}_{3}$ will be split according to the local meshsize near the discrete interface $F^{n}$ and far from it. Consider first $S \in \mathscr{S}_{F}^{n}=\left\{S \in \mathscr{S}^{n}\right.$ : $\left.S \cap F^{n} \neq \varnothing\right\}$, for which $h_{S}=O(\tau)$. The inequality (7.12) just used, now with $p=1$, leads also to

$$
\left\|\beta\left(U^{n}\right)-\Pi^{n} \beta\left(U^{n}\right)\right\|_{L^{1}(S)} \leq C \tau\left\|\nabla \Theta^{n}\right\|_{L^{1}(S)} .
$$

On the other hand, if $S \in \mathscr{S}^{n} \backslash \mathscr{S}_{F}^{n}$ we can exploit the further regularity of $\beta$, together with (4.10) and the constraint $h_{S} \leq \Lambda \tau^{1 / 2}$ for all $S \in \mathscr{S}^{n}$, to arrive at

$$
\begin{aligned}
\left\|\beta\left(U^{n}\right)-\Pi^{n} \beta\left(U^{n}\right)\right\|_{L^{1}(S)} & \leq C h_{S}^{2}\left\|D^{2} \beta\left(U^{n}\right)\right\|_{L^{1}(S)} \\
& \leq C L_{\beta^{\prime}} \tau\left\|\nabla U^{n}\right\|_{L^{2}(S)} \leq C L_{\beta^{\prime}} l_{\beta}^{-1} \tau\left\|\nabla \Theta^{n}\right\|_{L^{2}(S)}^{2},
\end{aligned}
$$

by virtue of a standard interpolation estimate. Hence, from (2.5), (6.2) and (6.4) we readily have

$$
\begin{aligned}
\left|\mathrm{II}_{3}\right| & \leq \sum_{n=1}^{m}\left(\sum_{S \in \mathcal{S}^{n}}\left\|\beta\left(U^{n}\right)-\Pi^{n} \beta\left(U^{n}\right)\right\|_{L^{1}(S)}\right) \int_{I^{n}}\left\|e_{u}(t)\right\|_{L^{\infty}(\Omega)} d t \\
& \leq C \tau \sum_{n=1}^{m} \tau\left(\left\|\nabla \Theta^{n}\right\|_{L^{1}(\Omega)}+\left\|\nabla \Theta^{n}\right\|_{L^{2}(\Omega)}^{2}\right) \leq C \tau .
\end{aligned}
$$

In summary, for term II we have obtained the lower bound

$$
\text { II } \geq\left(2 L_{\beta}\right)^{-1}\left\|e_{\theta}\right\|_{L^{2}\left(0, t^{m} ; L^{2}(\Omega)\right)}^{2}-C \tau .
$$

The analysis of term III on the right-hand side of (7.11) will be also split according to what happens within the refined region $\mathscr{R}^{n}$ (defined in $\S 2$ ) and out of it. More precisely, we decompose the integrals in III over all triangles $S \in \mathscr{S}_{R}^{n}:=\left\{S \in \mathscr{S}^{n}: S \subset \mathscr{R}^{n}\right\}$, where $h_{S}=O(\tau)$, and $S \in \mathscr{S}^{n} \backslash \mathscr{S}_{R}^{n}$, where $h_{S} \leq \Lambda \tau^{1 / 2}$. Inequality (7.1) then yields

$$
\begin{aligned}
|\mathrm{III}| & \leq \sum_{n=1}^{m} \sum_{S \in \mathscr{S}^{n}} \int_{S}\left|\left(U^{n}-\hat{U}^{n-1}\right) G^{n} e_{u}^{n}-\Pi^{n}\left[\left(U^{n}-\widehat{U}^{n-1}\right) G^{n} e_{u}^{n}\right]\right| d x \\
& \leq C \sum_{n=1}^{m}\left(\tau\left\|U^{n}-\hat{U}^{n-1}\right\|_{L^{2}(\Omega)}+\tau^{1 / 2}\left\|U^{n}-\widehat{U}^{n-1}\right\|_{L^{2}\left(\Omega \backslash \mathscr{R}^{n}\right)}\right)\left\|\nabla G^{n} e_{u}^{n}\right\|_{L^{2}(\Omega)} .
\end{aligned}
$$


Hence, applying (7.4) and the property $\left\|\nabla G^{n} \psi\right\|_{L^{2}(\Omega)} \leq\|\nabla G \psi\|_{L^{2}(\Omega)}$, together with (6.4) and (6.7), we get

$$
\begin{aligned}
|\mathrm{III}| & \leq \eta A_{m}+C \eta^{-1} \tau\left(\sum_{n=1}^{m}\left\|U^{n}-\widehat{U}^{n-1}\right\|_{L^{2}(\Omega)}^{2}+\sum_{n=1}^{m} \tau^{-1}\left\|U^{n}-\widehat{U}^{n-1}\right\|_{L^{2}\left(\Omega \backslash \mathscr{R}^{n}\right)}^{2}\right) \\
& \leq \eta A_{m}+C \eta^{-1} \tau,
\end{aligned}
$$

where $\eta>0$ is to be selected. The contribution due to IV can be handled by means of (2.5), (6.2), (6.8) and Lemma 7.1 as follows:

$$
|\mathrm{IV}| \leq \sum_{n=1}^{m}\left\|U^{n}-\widehat{U}^{n-1}\right\|_{L^{1}(\Omega)}\left\|\left(G-G^{n}\right) e_{u}^{n}\right\|_{L^{\infty}(\Omega)} \leq C \tau|\log \tau|^{7}
$$

Term $\mathrm{V}$ is easy to evaluate in view of $(5.10)$ and the fact that $U^{n-1} \neq \widehat{U}^{n-1}$ only whenever the mesh $\mathscr{S}^{n-1}$ changes. In fact, we have

$$
\begin{aligned}
|\mathrm{V}| & \leq \sum_{n=1}^{m}\left\|U^{n-1}-\hat{U}^{n-1}\right\|_{L^{2}(\Omega)}\left\|e_{u}^{n}\right\|_{H^{-1}(\Omega)} \\
& \leq A_{m}^{1 / 2} \sum_{n=1}^{m}\left\|U^{n-1}-\widehat{U}^{n-1}\right\|_{L^{2}(\Omega)} \leq C \tau^{1 / 2} A_{m}^{1 / 2} \leq \eta A_{m}+C \eta^{-1} \tau .
\end{aligned}
$$

It only remains to estimate the contribution due to the source $f$. To evaluate VI, we decompose it further as follows:

$$
\begin{aligned}
\mathrm{VI}= & \sum_{n=1}^{m}\left\langle\int_{I^{n}} f(\theta(t)) d t,\left(G-G^{n}\right) e_{u}^{n}\right\rangle \\
& +\sum_{n=1}^{m}\left\langle\int_{I^{n}}\left[f(\theta(t))-f\left(\boldsymbol{\Theta}^{n}\right)\right] d t, G^{n} e_{u}^{n}\right\rangle+\sum_{n=1}^{m} \tau\left\langle f\left(\boldsymbol{\Theta}^{n}\right)-f\left(\widehat{\boldsymbol{\Theta}}^{n-1}\right), G^{n} e_{u}^{n}\right\rangle \\
& +\sum_{n=1}^{m} \tau\left(\left\langle f\left(\hat{\boldsymbol{\Theta}}^{n-1}\right), G^{n} e_{u}^{n}\right\rangle-\left\langle f\left(\hat{\boldsymbol{\Theta}}^{n-1}\right), G^{n} e_{u}^{n}\right\rangle^{n}\right)=: \mathrm{VI}_{1}+\cdots+\mathrm{VI}_{4} .
\end{aligned}
$$

We have first

$$
\left|\mathrm{VI}_{1}\right| \leq C\|f(\theta)\|_{L^{\infty}\left(0, T ; L^{2}(\Omega)\right)} \max _{1 \leq n \leq m}\left\|\left(G-G^{n}\right) e_{u}^{n}\right\|_{L^{2}(\Omega)} \leq C \tau,
$$

as results from (2.4), (2.5), (6.2), and (7.6), as well as

$$
\begin{aligned}
\left|\mathrm{VI}_{2}\right| & \leq C \tau^{1 / 2} \sum_{n=1}^{m}\left\|e_{\theta}\right\|_{L^{2}\left(I^{n} ; L^{2}(\Omega)\right)}\left\|e_{u}^{n}\right\|_{H^{-1}(\Omega)} \\
& \leq \eta\left\|e_{\theta}\right\|_{L^{2}\left(0, t^{m} ; L^{2}(\Omega)\right)}^{2}+C \eta^{-1} \sum_{n=1}^{m} \tau\left\|e_{u}^{n}\right\|_{H^{-1}(\Omega)}^{2} .
\end{aligned}
$$

We next use (6.6) together with (2.4) to obtain

$$
\left|\mathrm{VI}_{3}\right| \leq C \tau \sum_{n=1}^{m}\left\|\boldsymbol{\Theta}^{n}-\widehat{\Theta}^{n-1}\right\|_{L^{2}(\Omega)}\left\|e_{u}^{n}\right\|_{H^{-1}(\Omega)} \leq \eta A_{m}+C \tau^{2}
$$


We then decompose $\mathrm{VI}_{4}$ further and apply (7.1) in conjunction with (5.14) to arrive at

$$
\begin{aligned}
\left|\mathrm{VI}_{4}\right| \leq & \tau\left|\sum_{n=1}^{m}\left\langle f\left(\widehat{\boldsymbol{\Theta}}^{n-1}\right)-\Pi^{n} f\left(\widehat{\boldsymbol{\Theta}}^{n-1}\right), G^{n} e_{u}^{n}\right\rangle\right| \\
& +\tau\left|\sum_{n=1}^{m}\left\langle\Pi^{n} f\left(\widehat{\boldsymbol{\Theta}}^{n-1}\right), G^{n} e_{u}^{n}\right\rangle-\left\langle\Pi^{n} f\left(\widehat{\boldsymbol{\Theta}}^{n-1}\right), G^{n} e_{u}^{n}\right\rangle^{n}\right| \\
\leq & \tau^{3 / 2} \sum_{n=1}^{m}\left\|\nabla \widehat{\boldsymbol{\Theta}}^{n-1}\right\|_{L^{2}(\Omega)}\left\|e_{u}^{n}\right\|_{H^{-1}(\Omega)} \leq \eta A_{m}+C \tau .
\end{aligned}
$$

A proper choice of $\eta$ finally allows terms $A_{m}$ and $\left\|e_{\theta}\right\|_{L^{2}\left(0, t^{m} ; L^{2}(\Omega)\right)}^{2}$ to be absorbed into the left-hand side of (7.11). Therefore, for all $1 \leq m \leq N$, we have obtained the estimate

$$
\max _{1 \leq n \leq m}\left\|e_{u}^{n}\right\|_{H^{-1}(\Omega)}^{2}+\left\|e_{\theta}\right\|_{L^{2}\left(0, t^{m} ; L^{2}(\Omega)\right)}^{2} \leq C \tau|\log \tau|^{7}+C \sum_{n=1}^{m} \tau\left\|e_{u}^{n}\right\|_{H^{-1}(\Omega)}^{2} .
$$

The rightmost term can be removed after applying the discrete Gronwall lemma. The desired estimate then follows from the property $u \in H^{1}\left(0, T ; H^{-1}(\Omega)\right) \subset$ $C^{0,1 / 2}\left(0, T ; H^{-1}(\Omega)\right)$, which can be used to replace $\max _{1 \leq n \leq m}\left\|e_{u}^{n}\right\|_{H^{-1}(\Omega)}$ by $\left\|e_{u}\right\|_{L^{\infty}\left(0, T ; H^{-1}(\Omega)\right)}$. The theorem is thus proved.

\section{Computational issues}

To conclude this paper, we present several numerical experiments and compare the proposed Adaptive Method (AM) with the Fixed Mesh Method (FMM). We also comment on some crucial computational issues. Full implementation details as well as many other relevant numerical tests will appear in Part II [16].

8.1. Implementation. Let $\mathbf{M}^{n}:=\left(\left\langle\chi_{i}^{n}, \chi_{j}^{n}\right\rangle^{n}\right)_{i, j=1}^{J^{n}}$ and $\mathbf{K}^{n}:=\left(\left\langle\nabla \chi_{i}^{n}, \nabla \chi_{j}^{n}\right\rangle\right)_{i, j=1}^{J^{n}}$ denote the mass matrix and the stiffness matrix, respectively. The equation (2.15) can then be written in matrix form as follows:

$$
\mathbf{M}^{n} U^{n}+\tau \mathbf{K}^{n} \Theta^{n}=\mathbf{M}^{n}\left(\widehat{U}^{n-1}+\tau f\left(\widehat{\boldsymbol{\Theta}}^{n-1}\right)\right),
$$

where we have identified piecewise linear functions with the vector of their nodal values. Since $\Theta^{n}=\Pi^{n}\left[\beta\left(U^{n}\right)\right]$, the algebraic system (8.1) is (strongly) nonlinear. However, as $\mathbf{M}^{n}$ is diagonal, (8.1) can be easily and efficiently solved by a nonlinear SOR method that is known to converge; see $[16,17,18]$. Based on a linear majorant, an approximate optimal relaxation parameter can be determined in advance so as to accelerate the convergence of this iterative method [16].

In order to implement (4.9) efficiently, an auxiliary (or superposed) uniform square mesh $\mathscr{Q}$ of size $\rho:=O\left(\tau^{1 / 2}\right)$ is used. Such a mesh is created and kept fixed from the beginning in such a way that $\bar{\Omega} \subset \bigcup_{R \in \mathscr{Q}} R$. We associate with 
each square $R$ a value of desired meshsize, defined as the minimum of the local parameters of $\S 4.1$ and $\S S 4.2$. This leads to the following piecewise constant function:

$$
\left.\check{\mathbf{h}}^{n}\right|_{R}:=\min _{\substack{S^{\prime} \in \mathscr{S}_{F}^{n}: \mathscr{E}_{S^{\prime} \cap R \neq \varnothing} \\ S \in \mathscr{S}_{0}^{n}, e \in \mathscr{E}_{0}^{n}: S \cap R \neq \varnothing, e \subset \partial S}}\left(\Lambda \tau^{1 / 2}, \hat{h}_{F_{S^{\prime}}}, \hat{h}_{e}, \hat{h}_{S}\right), \quad \forall R \in \mathscr{Q} .
$$

Such a function may be very oscillatory and thus not appropriate as a meshsize indicator. An efficient smcothing postprocess then constructs a piecewise constant function $\mathbf{h}^{n}$ satisfying the following compatibility property for all $R \in \mathscr{Q}$ :

$$
\left.\check{\mathbf{h}}^{n}\right|_{P} \geq\left.\mathbf{h}^{n}\right|_{R}, \quad \forall P \in \mathscr{Q}: \quad \operatorname{dist}(P, R) \leq\left.\mathbf{h}^{n}\right|_{R} .
$$

This is achieved, for instance, by defining $\mathbf{h}^{n}$ to be

$$
\left.\mathbf{h}^{n}\right|_{R}:=\min _{P \in \mathscr{Q}}\left(\max _{(}\left(\left.\check{\mathbf{h}}^{n}\right|_{P}, \operatorname{dist}(P, R)-\rho\right)\right), \quad \forall R \in \mathscr{Q} .
$$

The function $\mathbf{h}^{n}$ is then used by the automatic mesh generator of [19] to produce an admissible triangulation. In fact, by virtue of (8.3), we have $\lambda \tau \leq h_{S} \leq$ $\check{\mathbf{h}}^{n}(x) \leq \Lambda \tau^{1 / 2}$ for all $S \in \mathscr{S}^{n}$ and $x \in S$, which in turn implies (4.9). This topic is further discussed in [16].

Since several remeshing operations are to be performed during the solution process, mesh generator efficiency is a crucial issue. We used the mesh generator ADVFRONT of [19], which produces weakly acute meshes for general planaĩ domains with quasi-optimal computational complexity $O\left(\mathbf{S}^{n} \log \mathbf{S}^{n}\right)$, where $\mathbf{S}^{n}:=\operatorname{card}\left(\mathscr{S}^{n}\right)$. This is so because binary search techniques are employed on suitable quadtree structured data to update the advancing front. Similar ideas were applied to determine $\widehat{U}^{n-1}$, the interpolant of $U^{n-1}$ in $\mathscr{S}^{n}$. More specifically, this crucial interpolation process requires a computational labor of $O\left(\left(\mathbf{S}^{n-1}+J^{n}\right) \log J^{n}\right)$, which is also nearly optimal; recall that $J^{n}$ is the numler of nodes of $\mathscr{S}^{n}$. Such techniques are essential for the Adaptive Method to be competitive. See [16] for more details.

8.2. Degrees of freedom. Suppose that there is no discrete mushy region and any discrete interface $F^{n}$ is a polygonal curve with finite length uniformly in $\tau$, which in turn is consistent with (3.13). Then the refined region $\mathscr{R}^{n}$ is just a strip $O\left(\tau^{1 / 2}\right)$-wide around $F^{n}$. Since the local meshsize is $O(\tau)$, the number of triangles within $\mathscr{R}^{n}$ is $O\left(\tau^{-3 / 2}\right)$. Except possibly for a small transition region, triangles outside of $\mathscr{R}^{n}$ are $O\left(\tau^{1 / 2}\right)$. Consequently, the number of triangles outside of $\mathscr{R}^{n}$ becomes $O\left(\tau^{-1}\right)$; so the required computational labor in $\mathscr{R}^{n}$ dominates! Hence, the number of spatial degrees of freedom (DOF) for every $\operatorname{mesh} \mathscr{S}^{n}$ is

$$
\mathrm{DOF}=O\left(\tau^{-3 / 2}\right)
$$

for a global accuracy $O\left(\tau^{1 / 2}\right)$. This quantity compares quite favorably with similar ones for practical methods involving a single quasi-uniform mesh and is 
reflected in Tables 8.1-8.3. In fact, in that case $h_{S}=O(\tau)$ for all $S \in \mathscr{S}$ and so $\mathrm{DOF}=O\left(\tau^{-2}\right)$ for the same global accuracy $[4,17,24]$. We also stressed that even with mushy regions, we need fewer DOF. A relevant example is fully discussed in [16].

Consider now the Fixed Mesh Method with preliminary regularization [8, $12,13,17]$. Assuming that a global nondegeneracy property is valid, a result similar to (8.5) can be obtained [17], but at the expense of a much worse local approximation quality, as numerical evidence indicates $[16,17,18]$. Such a local property is extremely important in determining interfaces and explains the much better resolution associated with the present method. We also underline that the example below does not satisfy a global nondegeneracy property.

8.3. Numerical experiments. To illustrate the superior performance of our Adaptive Method with respect to fixed mesh techniques, we have chosen the severe test below. It is a classical two-phase Stefan problem with an interface that moves up and down. The exact temperature is given by the following expression:

$$
\theta(x, y, t):= \begin{cases}0.75\left(r^{2}-1\right), & r<1, \\ \left(1.5-\alpha^{\prime}(t) \sin \varphi\right)(r-1), & r \geq 1,\end{cases}
$$

where $r:=\left(x^{2}+(y-\alpha(t))^{2}\right)^{1 / 2}, \alpha(t):=0.5+\sin (1.25 t), \sin \varphi:=(y-\alpha(t)) / r$, $\Omega:=(0,5) \times(0,5)$ and $T:=\pi / 1.25$. Dirichlet boundary conditions are imposed at $y=0, y=5$ and $x=5$, and a homogeneous Neumann condition is prescribed at $x=0$. Since the exact interface $I(t)$ is a circle with center $(0, \alpha(t))$ and radius 1 , the velocity $V(x, y, t)$ normal to $I(t)$ at $(x, y)$ exhibits a significant variation along the front, which makes this example an extremely difficult test for our numerical method. Moreover, since $V(x, y, t)$ vanishes at both $(x, y, T / 2)$ and $(1, \alpha(t), t)$, and is thus very small nearby, this test constitutes a fair measure of robustness under degenerate situations.

Several numerical experiments were performed with both our Adaptive Method (with and without fixed triangles as in $\S 4.2$ and $\S S 4.1$ ) and the Fixed Mesh Method [3, 4, 8, 12, 13, 17, 24]. For the latter, the constant of proportionality between $\tau$ and the (uniform) meshsize was chosen so as to minimize $\left\|e_{\theta}\right\|_{L^{2}(Q)}$ for a desired number of DOF, where $Q:=\Omega \times(0, T)$. The various constants introduced in $\S \S 4, \mathrm{~S} 4,8$ are as follows: $\lambda=1.5, \Lambda=5, M=5$, $\rho=0.75 \sqrt{\tau}, \mu_{1}=2.22, \mu_{2}=5.59$, average value of $\mu_{3} \approx 1.2, \mu_{1}^{\star}=\mu_{2}^{\star}=3.5$, $\mu_{3}^{-}=0.5, \mu_{3}^{+}=2, C_{1}=\left\|D^{2} \theta_{0}\right\|_{L^{\infty}(\Omega)}, C_{2}=3\left\|\nabla \theta_{0}\right\|_{L^{\infty}(\Omega)}, C_{3}=2$ length $\left(I_{0}\right)$, $C_{4}=C_{3} \lambda$.

Results are reported in Tables 8.1-8.3, where we have employed the following notation: $\mathbf{N}:=$ number of (uniform) time steps, $\mathbf{J}:=$ average number of nodes, $\mathbf{S}_{R}:=$ average number of triangles within the refined region, $\mathbf{S}_{P}:=$ average number oî triangles in the rest of $\Omega, \mathbf{S}:=$ total number of triangles (uniform mesh), C := number of mesh changes (and number of computed solutions rejected, if any), CPU := CPU time in seconds (on a vaX 
TABLE 8.1

Adaptive method (with fixed triangles)

\begin{tabular}{|c|c|c|c|c|c|c|c|c|c|}
\hline $\mathbf{N}$ & $\mathbf{J}$ & $\mathbf{S}_{R}$ & $\mathbf{S}_{P}$ & $\mathbf{C}$ & $\mathbf{E}_{\theta}^{2}$ & $\mathbf{E}_{u}^{2}$ & $\mathbf{E}_{\theta}^{\infty}$ & $\mathbf{E}_{I}^{\infty}$ & $\mathbf{C P U}$ \\
\hline 40 & 319 & 496 & 148 & $4(1)$ & 16.1 & 35.4 & 11.3 & 5.37 & 58 \\
60 & 593 & 957 & 233 & $4(1)$ & 10.5 & 29.1 & 6.65 & 4.44 & 132 \\
80 & 875 & 1430 & 328 & 4 & 7.54 & 24.2 & 5.23 & 3.45 & 224 \\
120 & 1413 & 2320 & 510 & 5 & 5.34 & 22.7 & 3.93 & 2.60 & 546 \\
160 & 2089 & 3504 & 679 & $6(1)$ & 4.07 & 19.6 & 3.14 & 2.08 & 111 \\
240 & 3620 & 6201 & 1042 & $8(1)$ & 2.82 & 17.7 & 2.41 & 1.65 & 2998 \\
\hline
\end{tabular}

TABLE 8.2

Adaptive method (without fixed triangles)

\begin{tabular}{|c|c|c|c|c|c|c|c|c|c|}
\hline $\mathbf{N}$ & $\mathbf{J}$ & $\mathbf{S}_{R}$ & $\mathbf{S}_{P}$ & $\mathbf{C}$ & $\mathbf{E}_{\theta}^{2}$ & $\mathbf{E}_{u}^{2}$ & $\mathbf{E}_{\theta}^{\infty}$ & $\mathbf{E}_{I}^{\infty}$ & $\mathbf{C P U}$ \\
\hline 40 & 339 & 536 & 144 & 3 & 18.8 & 37.0 & 10.7 & 5.83 & 50 \\
60 & 592 & 957 & 231 & 4 & 11.3 & 28.8 & 7.42 & 5.01 & 126 \\
80 & 818 & 1306 & 334 & 5 & 7.59 & 26.1 & 5.17 & 3.26 & 235 \\
120 & 1406 & 2306 & 510 & 6 & 5.73 & 23.3 & 3.92 & 2.66 & 583 \\
160 & 2110 & 3563 & 662 & 6 & 4.60 & 20.8 & 3.44 & 2.32 & 1068 \\
240 & 3631 & 6245 & 1023 & 8 & 2.99 & 18.5 & 2.58 & 1.72 & 2916 \\
\hline
\end{tabular}

TABLE 8.3

Fixed mesh method

\begin{tabular}{|c|c|c|c|c|c|c|c|}
\hline $\mathbf{N}$ & $\mathbf{J}$ & $\mathbf{S}$ & $\mathbf{E}_{\theta}^{2}$ & $\mathbf{E}_{u}^{2}$ & $\mathbf{E}_{\theta}^{\infty}$ & $\mathbf{E}_{I}^{\infty}$ & $\mathbf{C P U}$ \\
\hline 50 & 448 & 942 & 15.9 & 39.7 & 20.8 & 13.8 & 37 \\
75 & 1017 & 2104 & 10.5 & 30.4 & 15.3 & 9.40 & 113 \\
100 & 1812 & 3718 & 7.81 & 26.8 & 12.4 & 6.88 & 292 \\
150 & 4107 & 8356 & 5.57 & 22.3 & 7.78 & 5.65 & 919 \\
200 & 7361 & 14912 & 4.52 & 18.6 & 6.31 & 3.53 & 2264 \\
\hline
\end{tabular}

8530, vMS 4.6) and $\mathbf{E}_{\theta}^{2}:=\left\|e_{\theta}\right\|_{L^{2}(Q)}, \quad \mathbf{E}_{u}^{2}:=\left\|e_{u}\right\|_{L^{2}(Q)}, \mathbf{E}_{\theta}^{\infty}:=\left\|e_{\theta}\right\|_{L^{\infty}(Q)}$, $\mathbf{E}_{I}^{\infty}:=\max _{1 \leq n \leq \mathrm{N}} \operatorname{dist}\left(I(n \tau), F^{n}\right)$, where the errors are scaled by $10^{2}$. Moreover, $\|\theta\|_{L^{2}(Q)} \approx 33.16,\|\theta\|_{L^{\infty}(Q)} \approx 13.38,\|u\|_{L^{2}(Q)} \approx 39.81$, with an error of one unit in the fourth digit.

In light of these (partial) results, we can certainly claim a superior performance of the Adaptive Method in that it requires less computational labor, say CPU, for a desired global accuracy. Moreover, the proposed local refinement strategies of $\S 4.2$ and $\S S 4.1$, with and without fixed triangles, perform quite similarly. The $L^{2}$-error for temperature $\mathbf{E}_{\theta}^{2}$ behaves linearly in $\tau$, thus much better than predicted. We also have a (linear) pointwise error $\mathbf{E}_{\theta}^{\infty}$ that 

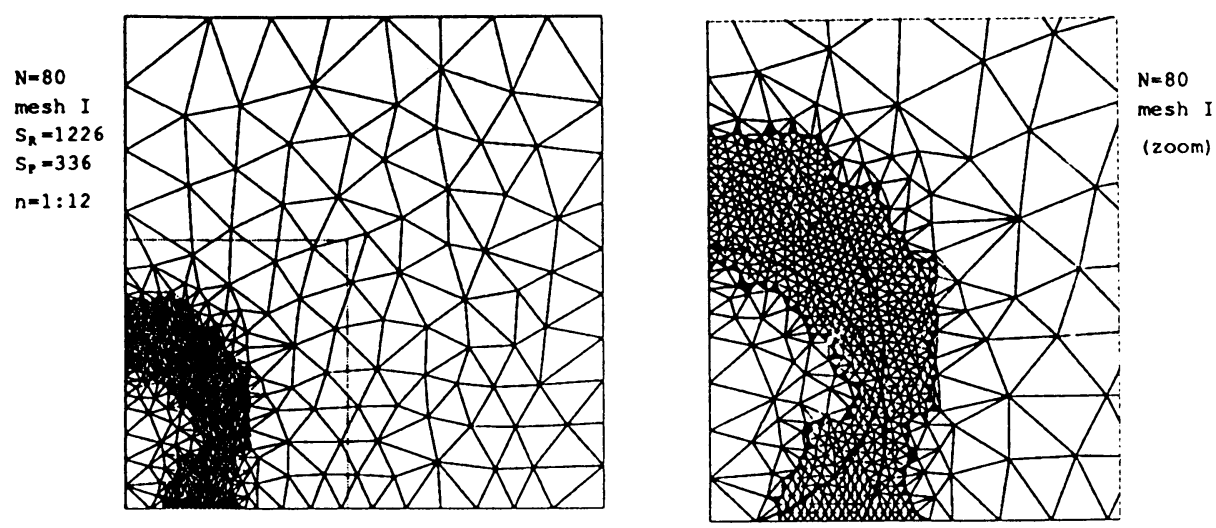

FIGURE 8.1

$\mathrm{AM}, \mathbf{N}=80, \mathscr{S}_{F} \cup \mathscr{S}_{B}$ free: Mesh $\mathrm{I}$ and zoom of refined region

is far from being theoretically explainable. The same happens with $\mathbf{E}_{u}^{2}$ and $\mathbf{E}_{I}^{\infty}$. The improvement gained in $L^{\infty}$ is clearly more pronounced than that in $L^{2}$. The free boundary is located within one single element, thus confirming the aforementioned local approximation quality. Therefore, we have a practical $O(\tau)$-rate of convergence in distance for interfaces, the best one can hope for. Since approximability and nondegeneracy are tied together [14], it is worth noting that the nondegeneracy property is not uniform in the present case.

We finally conclude with several pictures corresponding to the case $\mathbf{N}=80$ without fixed triangles. The first mesh together with the corresponding Refined Region is depicted in Figure 8.1. The boundary of the Refined Region, called RED ZONE, appears blackened in Figure $8.1 \mathrm{~b}$, which also shows the first and last discrete interfaces computed with mesh I. Note that the last interface has escaped from the Refined Region and should thus be discarded as indicated in $\S 4.2$. To avoid rejecting a computed solution owing to failure of the free boundary location test, a more flexible strategy for the case without fixed triangles has been designed in [16]. The remaining meshes, generated automatically by ADVFRONT are illustrated in Figure 8.2. Observe the proper grading produced by ADVFRONT. We see how the refined region moves up and down accompanying the interface motion. Note that even in the upmost position, when the interface is motionless, the proposed strategy is successful. Figure 8.3 is a zoom of both the exact and discrete interfaces for a number of time steps. The agreement between these curves is quite remarkable as compared with the local meshsize. Part of the RED zONE, blackened triangles, can be seen in the second zoom as well. The exact and discrete interfaces obtained with the FMM for $\mathbf{N}=100$ are depicted in Figure 8.4. It is worth comparing this picture with Figure 8.3: the interface location is drastically improved by the AM. 

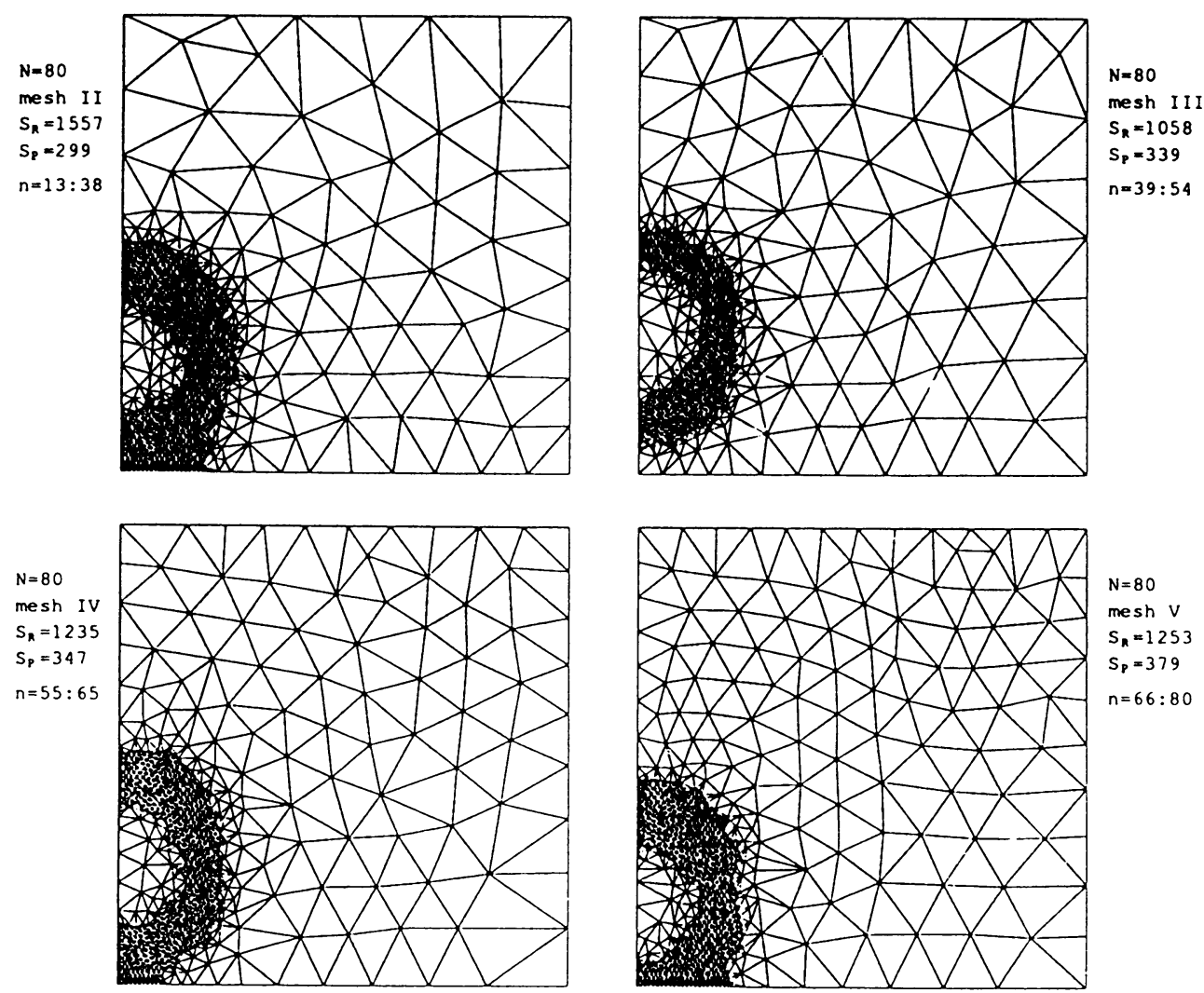

FIGURE 8.2

$\mathrm{AM}, \mathbf{N}=80, \mathscr{S}_{F} \cup \mathscr{S}_{B}$ free: Consecutive meshes
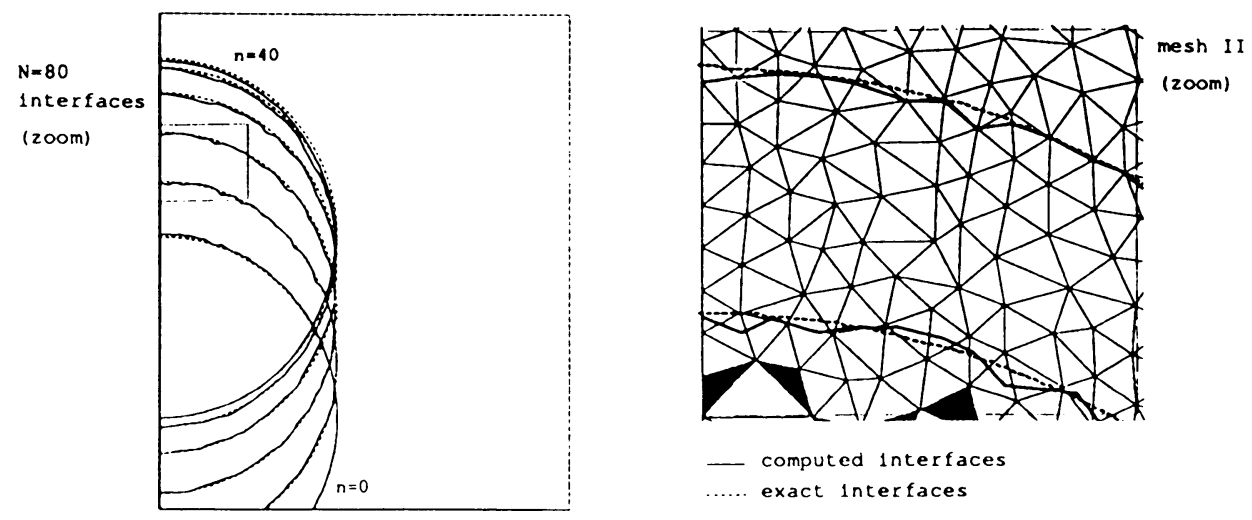

Figure 8.3

$\mathrm{AM}, \mathbf{N}=80, \mathscr{S}_{F} \cup \mathscr{S}_{B}$ free: Interfaces at $n=8 k \quad(0 \leq k \leq 5)$; zoom of Mesh II 

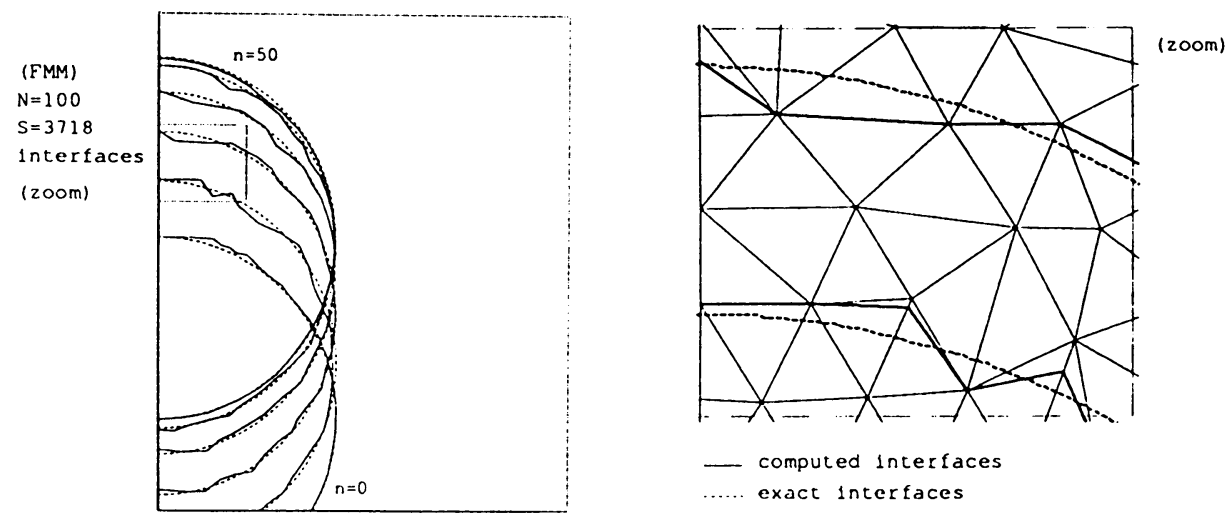

FIGURE 8.4

FMM, $\mathbf{N}=100:$ Interfaces at $n=10 k \quad(0 \leq k \leq 5)$; zoom of the mesh

\section{BIBLIOGRAPHY}

1. I. Babuška, O. C. Zienkiewicz, J. Gago, and E. R. de A. Oliveira, Accuracy estimates and adaptive refinements in finite element computations, Wiley, Chichester, 1986.

2. P. G. Ciarlet, The finite element method for elliptic problems, North-Holland, Amsterdam, 1978.

3. J. F. Ciavaldini, Analyse numérique d'un problème de Stefan à deux phases par une méthode d'éléments finis, SIAM J. Numer. Anal. 12 (1975), 464-487.

4. C. M. Elliott, Error analysis of the enthalpy method for the Stefan problem, IMA J. Numer. Anal. 7 (1987), 61-71.

5. K. Eriksson and C. Johnson, Adaptive finite element methods for parabolic problems I: A linear problem, preprint 31, University of Göteborg, 1988.

6. A. Friedman, The Stefan problem in several space variables, Trans. Amer. Math. Soc. 133 (1968), 51-87.

7. P. Grisvard, Eliiptic problems in non-smooth domains, Pitman, Boston, 1985.

8. J. W. Jerome and M. Rose, Error estimates for the multidimensional two-phase Stefan problem, Math. Comp. 39 (1982), 377-414.

9. R. Löhner, Some useful data structures for the generation of unstructured meshes, Comm. Appl. Numer. Methods 4 (1988), 123-135.

10. E. Magenes, Problemi di Stefan bifase in più variabili spaziali, Matematiche 36 (1981), 65-108.

11. E. Magenes, C. Verdi, and A. Visintin, Theoretical and numerical results on the two-phase Stefan problem, SIAM J. Numer. Anal. 26 (1989), 1425-1438.

12. R. H. Nochetto, Error estimates for two-phase Stefan problems in several space variables, I: Linear boundary conditions, Calcolo 22 (1985), 457-499.

13. _ـ Error estimates for multidimensional singular parabolic problems, Japan J. Appl. Math. 4 (1987), 111-138.

14. __ Numerical methods for free boundary problems, Free Boundary Problems: Theory and Applications II (K.-H. Hoffmann and J. Sprekels, eds.), Res. Notes in Math. 186, Pitman, London, 1990, pp. 555-566. 
15. R. H. Nochetto, M. Paolini, and C. Verdi, Local mesh refinements for two-phase Stefan problems in two space variables, Mathematical Models for Phase Change Problems (J. F. Rodrigues, ed.), Birkhäuser Verlag, Basel, 1989, pp. 261-286.

16. __ An adaptive finite element method for two-phase Stefan problems in two space dimensions. Part II: Implementation and numerical experiments, SIAM J. Sci. Statist. Comput. 1991 (to appear).

17. R.H. Nochetto and C. Verdi, Approximation of degenerate parabolic problems using numerical integration, SIAM J. Numer. Anal. 25 (1988), 784-814.

18. M. Paolini, G. Sacchi and C. Verdi, Finite element approximations of singular parabolic problems, Internat. J. Numer. Methods. Engrg. 26 (1988), 1989-2007.

19. M. Paolini and C. Verdi, An automatic triangular mesh generator for planar domains, Riv. Inform. 20 (1990), 251-267.

20. J. C. W. Rogers and A. E. Berger, Some properties of the nonlinear semigroup for the problem $u_{t}-\Delta f(u)=0$, Nonlinear Anal. 8 (1984), 909-939.

21. A. H. Schatz and L. B. Wahlbin, Maximum norm estimates in the finite element method on plane polygonal domains. Part 1, Math. Comp. 32 (1978), 73-109.

22. __ Maximum norm estimates in the finite element method on plane polygonal domains. Part 2, Refinements, Math. Comp. 33 (1979), 465-492.

23. $\ldots$, On the quasi-optimality in $L^{\infty}$ of the $H_{0}^{1}$-projection into finite element spaces, Math. Comp. 38 (1982), 1-22.

24. C. Verdi, Optimal error estimates for an approximation of degenerate parabolic problems, Numer. Funct. Anal. Optim. 9 (1987), 657-670.

Department of Mathematics and Institute for Physical Science and Technology, University of Maryland, College Park, MD 20742

Istituto di ANAlisi Numerica del CNR, 27100 Pavia, Italy

Dipartimento di Meccanica Strutturale, Università di Pavia and Istituto di ANalisi Numerica del CNR, 27100 Pavia, Italy 


\title{
Supplement to
}

\section{AN ADAPTIVE FINITE ELEMENT METHOD FOR TWO-PHASE STEFAN PROBLEMS IN TWO SPACE DIMENSIONS. PART I: STABILITY AND ERROR ESTIMATES}

\author{
R. H. NOCHETTO† M. PAOLINI $\ddagger$ AND C. VERDI $\sharp$
}

\section{S1. Supplement to Section 5.}

Lemma 5.1. For all regular meshes $\mathcal{S}$ there exists a $C^{\infty}$-function $h: \bar{\Omega} \rightarrow \mathbf{R}^{+}$satisfying

$$
D^{k} h(x)=O\left(h_{S}^{1-k}\right), \quad \forall x \in S \in \mathcal{S}, k \geq 0 .
$$

Proof. For each $S \in \mathcal{S}$ there exists a (finite) covering $\left\{B_{i}\right\}$ of $S$ such that card $\left\{B_{i}\right\}=$ $O(1)$, where $B_{i}:=B\left(x_{i}, r_{i}\right), x_{i} \in S$ and $r_{i}:=a h_{S} / 2$; thus (5.2) yields $\bar{B}_{i}:=B\left(x_{i}, 2 r_{i}\right) \subset$ $\tilde{S}$. By virtue of (5.3), this gives rise to a covering $\left\{B_{i}\right\}_{i=1}^{I}$ of $\bar{\Omega}$ satisfying $b_{S}:=$ card $\left\{B_{i}: S \cap \bar{B}_{i} \neq \emptyset\right\}=O(1)$ for all $S \in \mathcal{S}$ (nonoverlapping property !). Let $\delta_{0} \in C_{0}^{\infty}(B(0,1)$ ) satisfy $\int_{\mathbf{R}^{2}} \delta_{0}=1,0 \leq \delta_{0} \leq 1, \delta_{0}(x)=1$ for all $x \in B(0,1 / 2)$ and $\delta_{0}$ is radially symmetric (mollifier function). Set $\mu_{i}(x):=\delta_{0}\left(\left(x-x_{i}\right) /\left(2 r_{i}\right)\right)$ for all $x \in \bar{\Omega}$ and note that

$$
\operatorname{supp} \mu_{i}=\bar{B}_{i} \subset \tilde{S}, \quad 1 \leq \sum_{i=1}^{I} \mu_{i}(x) \leq b, \quad \forall x \in \bar{\Omega},
$$

where $b$ depends only on the regularity of $\mathcal{S}$; we certainly have $b<b_{S}=O(1)$ for all $S \in \mathcal{S}$. Define now $h \in C^{\infty}(\bar{\Omega})$ to be

$$
h(x):=\frac{a}{b} \sum_{i=1}^{I} r_{i} \mu_{i}(x), \quad \forall x \in \bar{\Omega} .
$$

Since $\rho_{S^{\prime}}<h_{S}$ for all $S^{\prime} \in \mathcal{S}_{S}$, it is easily seen from (5.1) that, if $x_{i} \in S$, then

$$
\frac{a^{2}}{b} r_{i}=\frac{a^{3}}{2 b} h_{S} \leq \frac{a^{2}}{2 b} \min _{S^{\prime} \in \mathcal{S}_{S}} \rho_{S^{\prime}} \leq h(x) \leq \frac{a}{2} \max _{S^{\prime} \in \mathcal{S}_{S}} \rho_{S^{\prime}}<\frac{a}{2} h_{S}=r_{i}, \quad \forall x \in B_{i} .
$$

Hence $B(x, h(x)) \subset \bar{B}_{i}(\subset \tilde{S})$ for all $x \in B_{i}$. Since

$$
D^{k} h(x)=\frac{a}{b} \sum_{i=1}^{I} r_{i} D^{k} \mu_{i}(x)=C \sum_{i=1}^{I} r_{i}^{1-k} D^{k} \delta_{0}\left(\left(x-x_{i}\right) /\left(2 r_{i}\right)\right), \quad \forall x \in \Omega,
$$

the desired result easily follows from (S1.2).

†Department of Mathematics and Institute for Physical Science and Technology, University of Maryland, College Park, MD 20742 USA.

†stituto di Analisi Numerica del CNR, 27100 Pavia, Italy.

\#Dipartimento di Meccanica Strutturale, Università di Pavia and Istituto di Analisi Numerica del CNR, 27100 Pavia, Italy. 

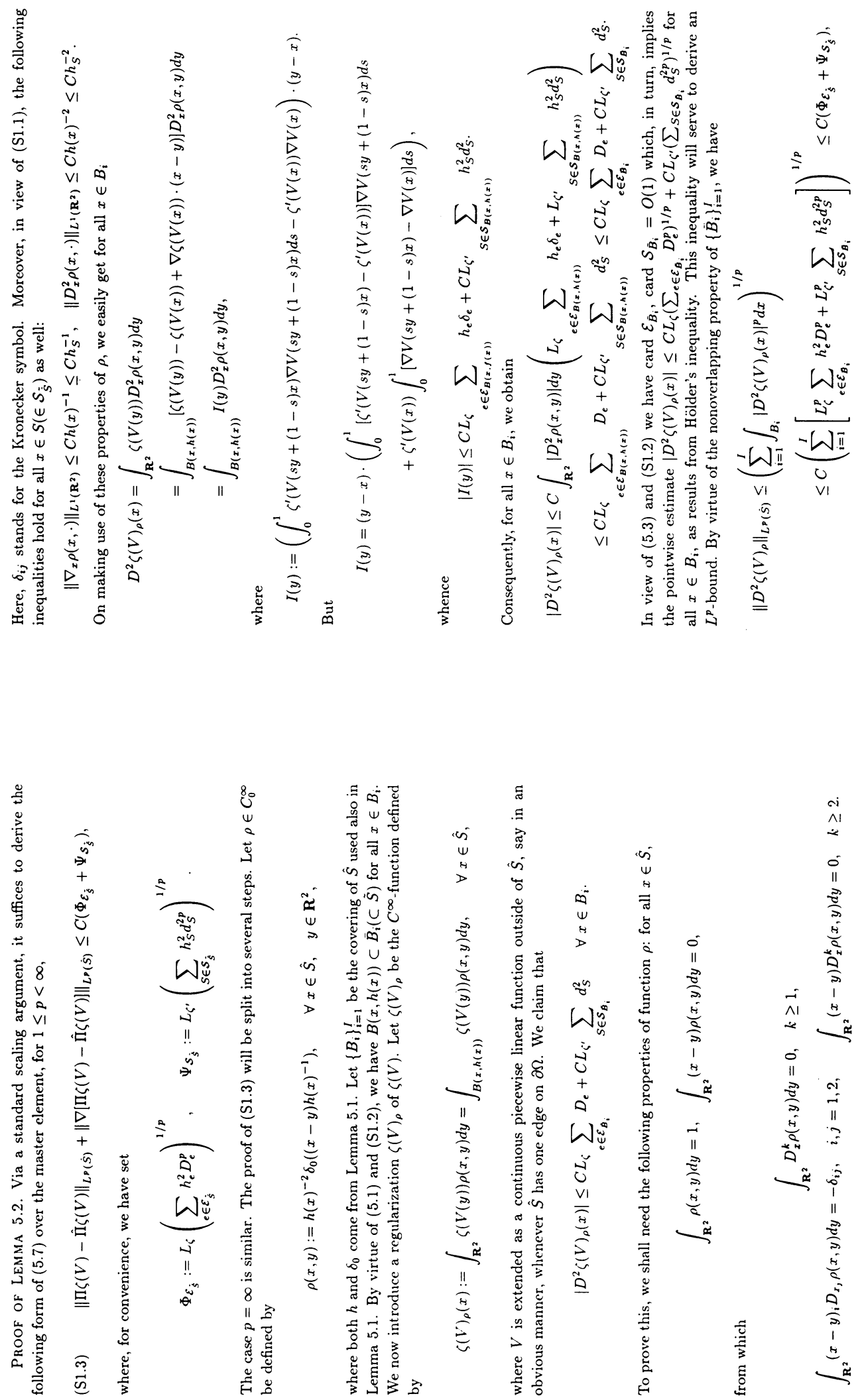

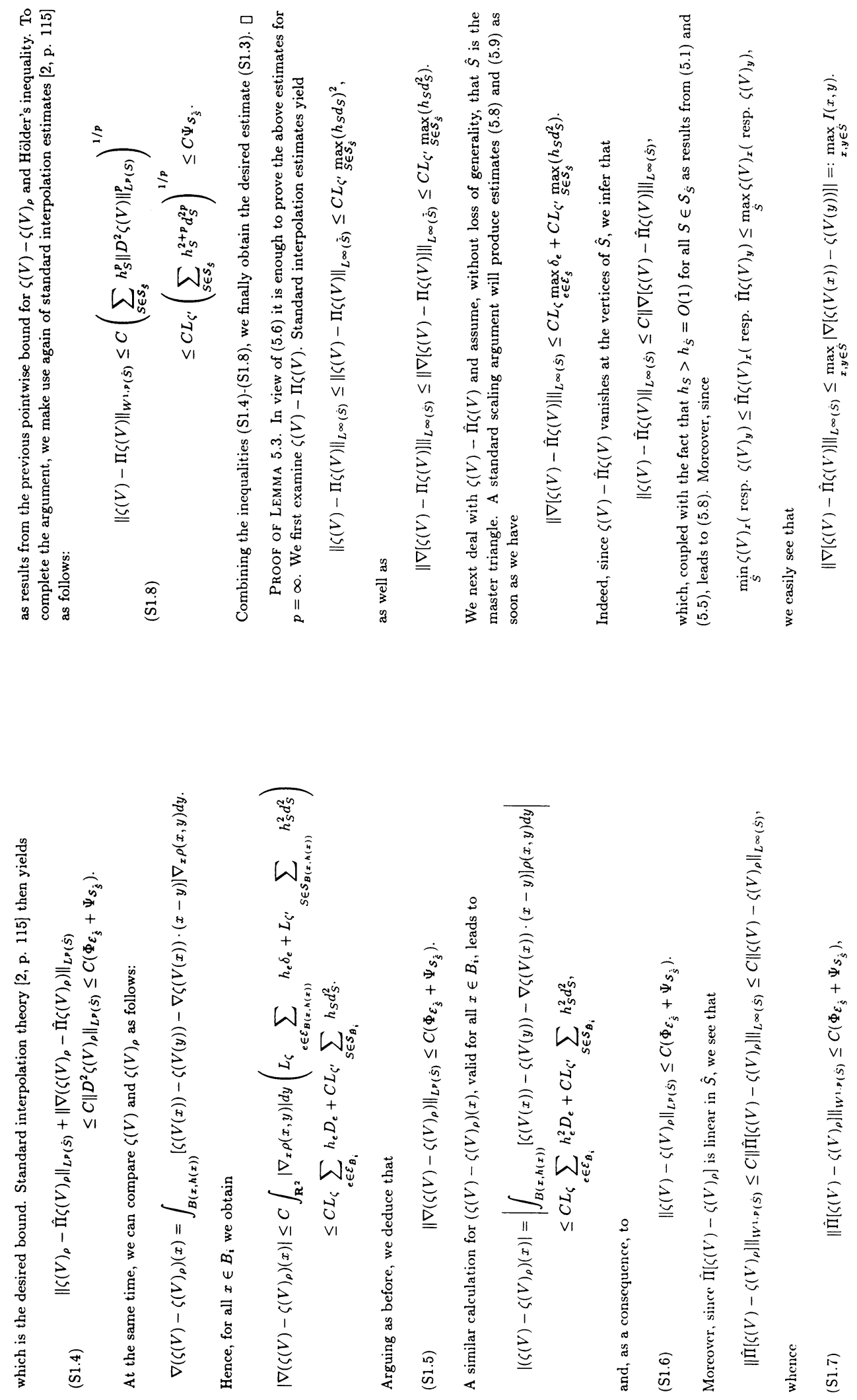

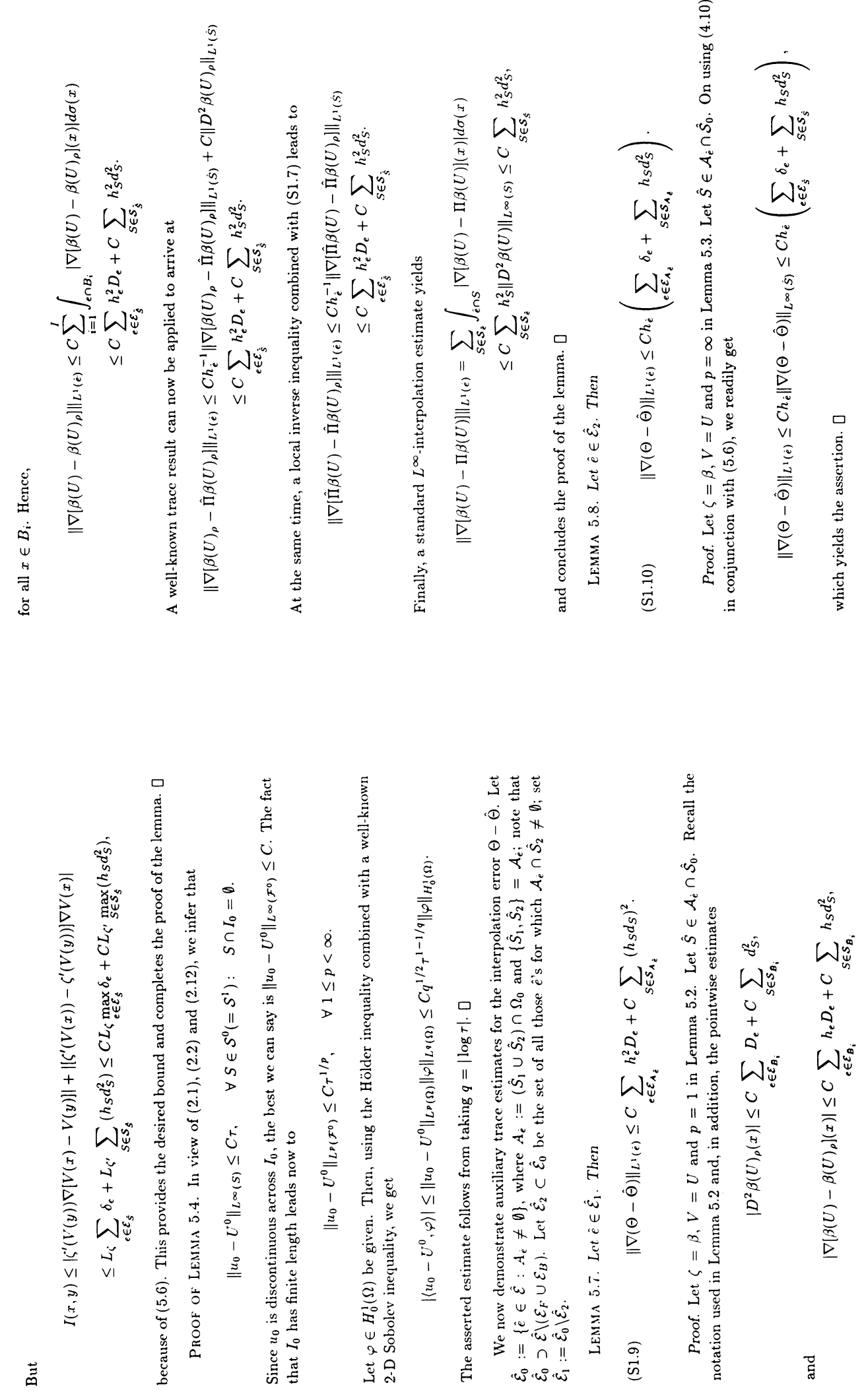

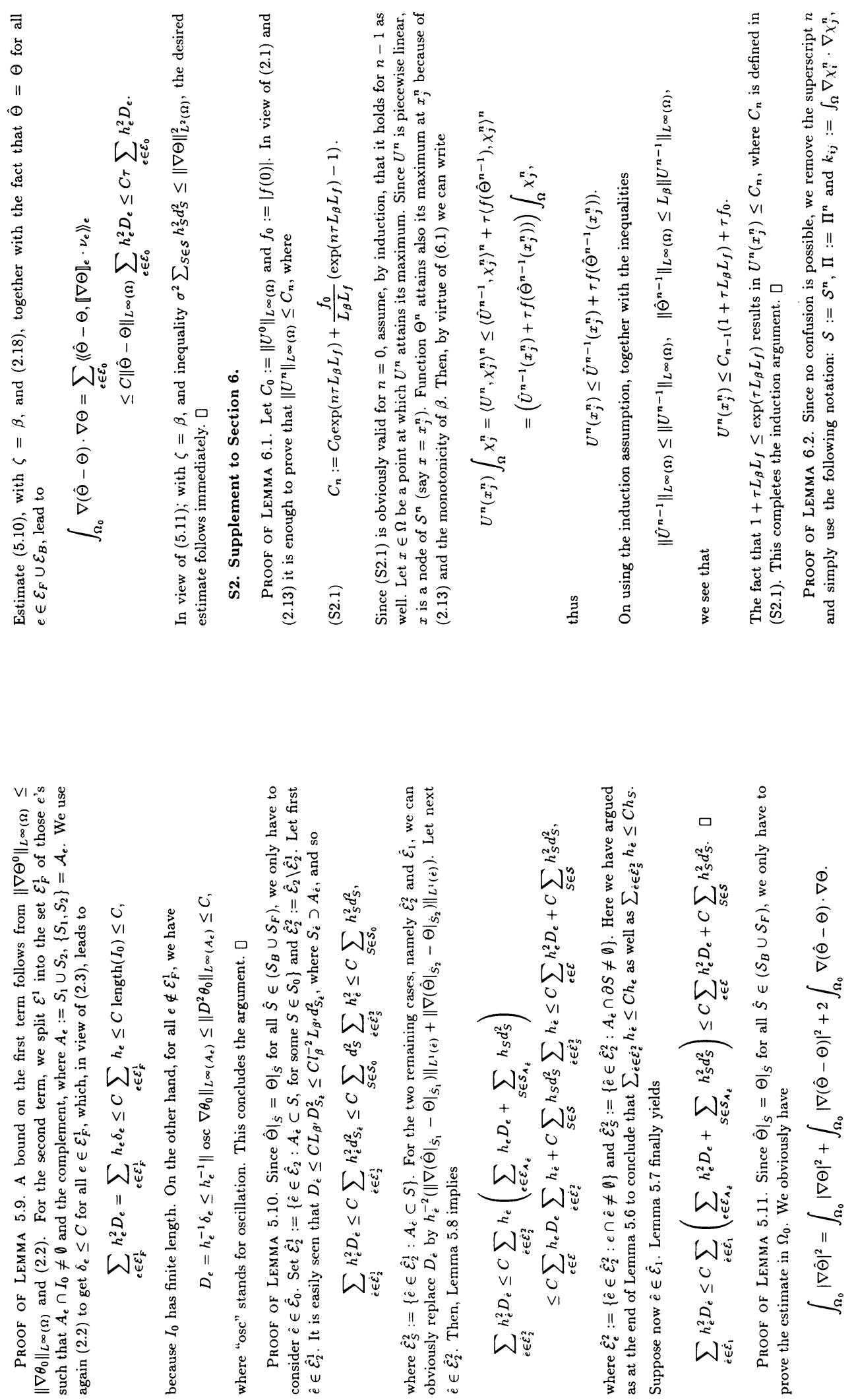

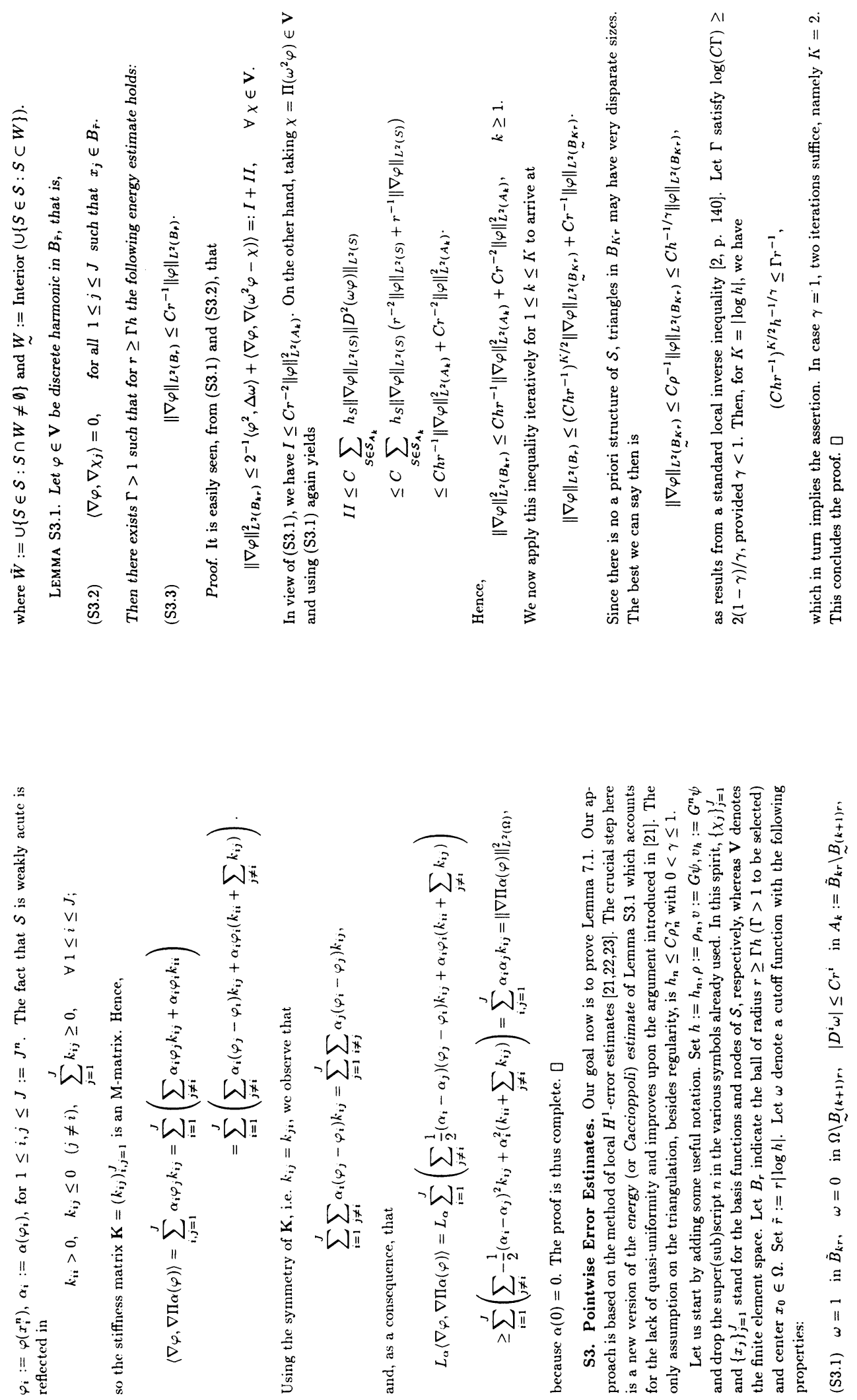


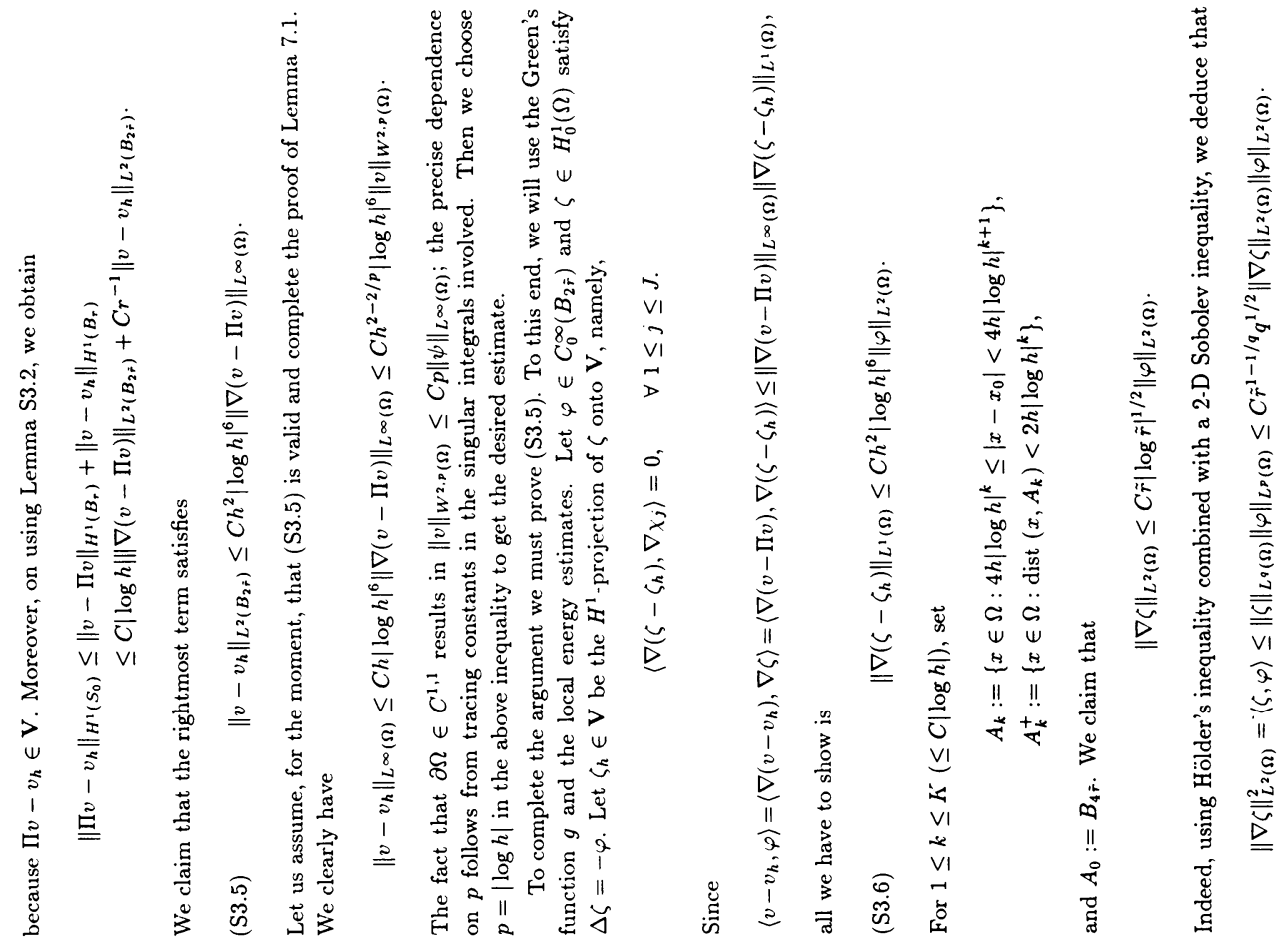

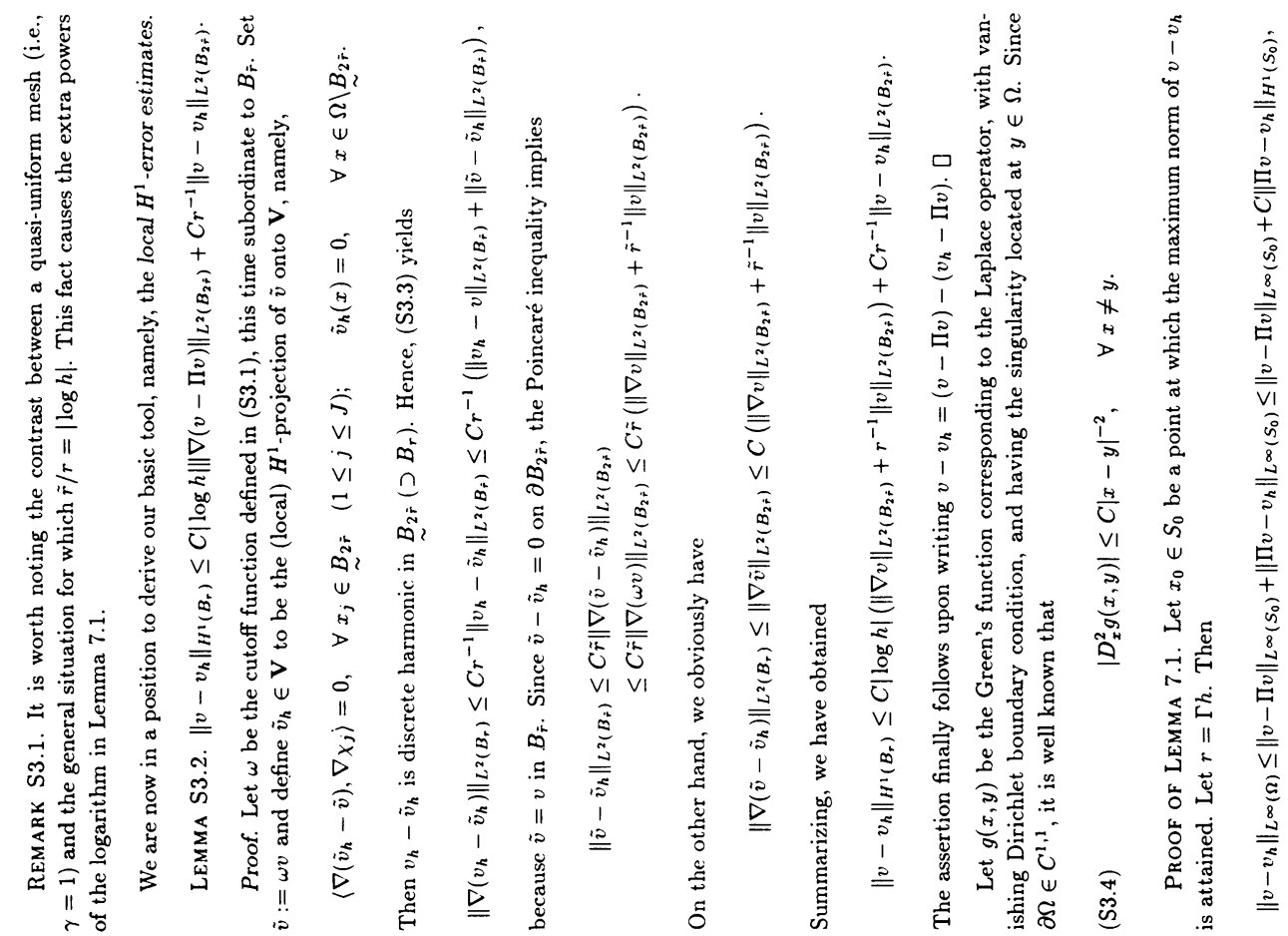



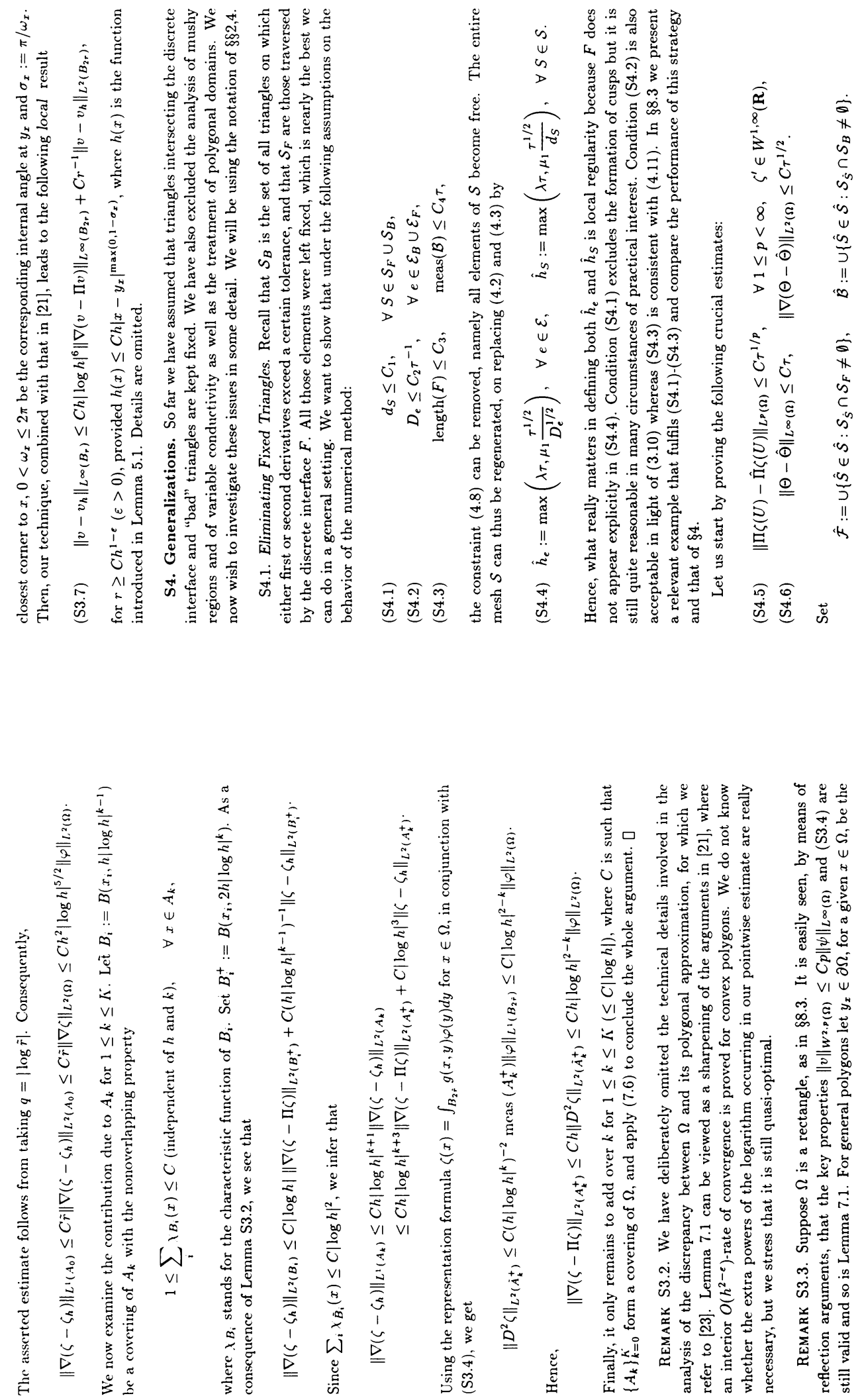

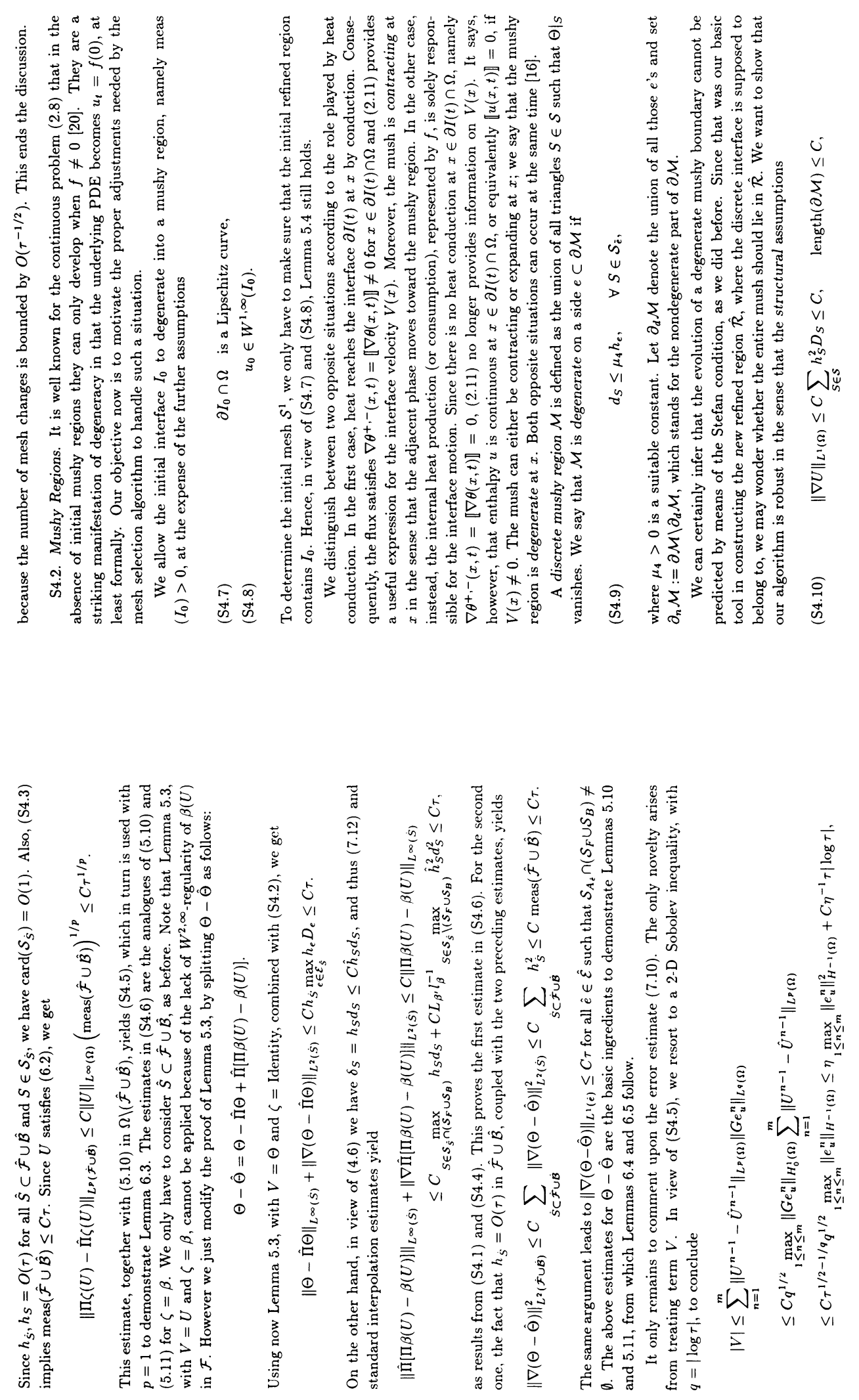


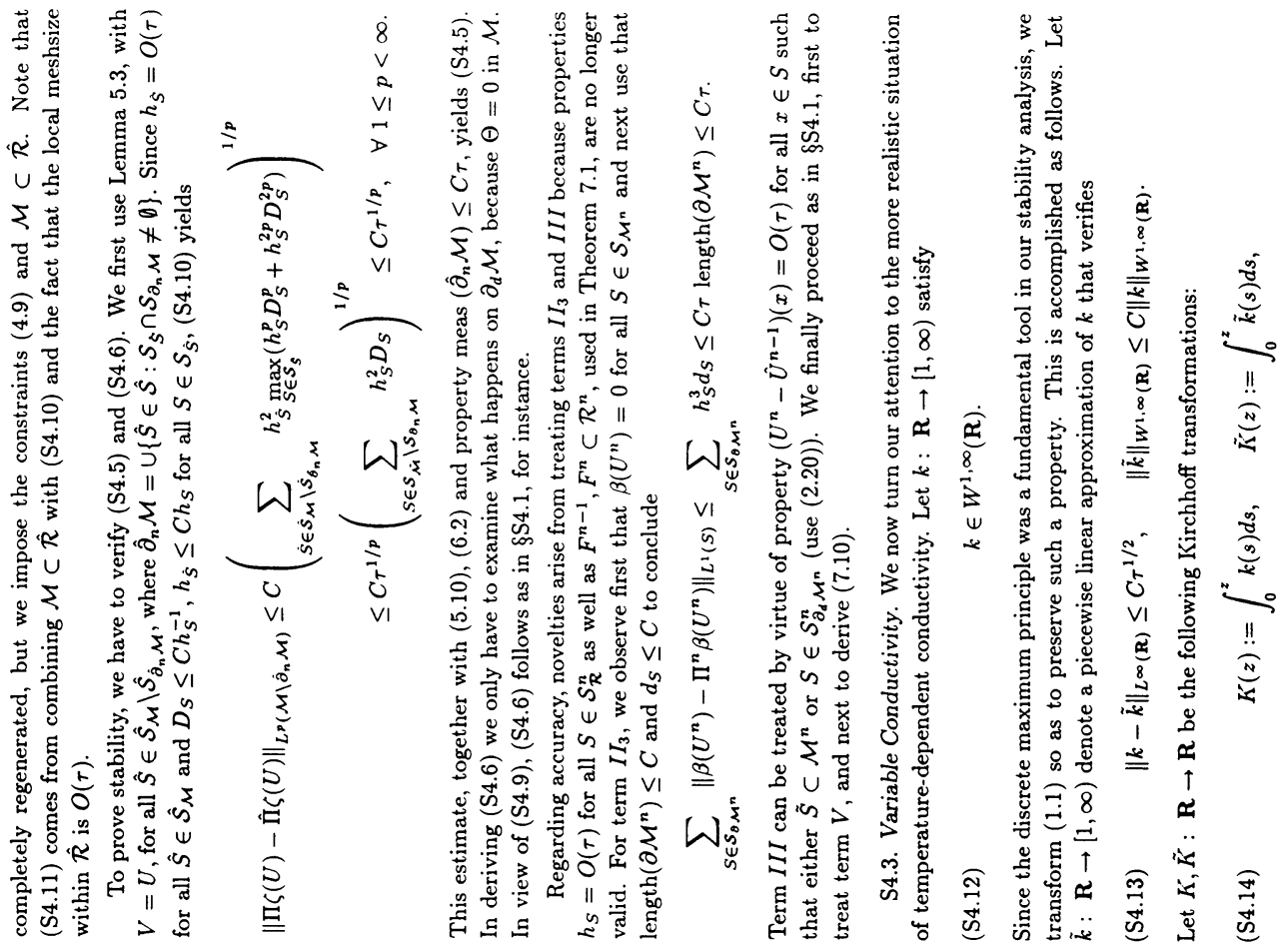

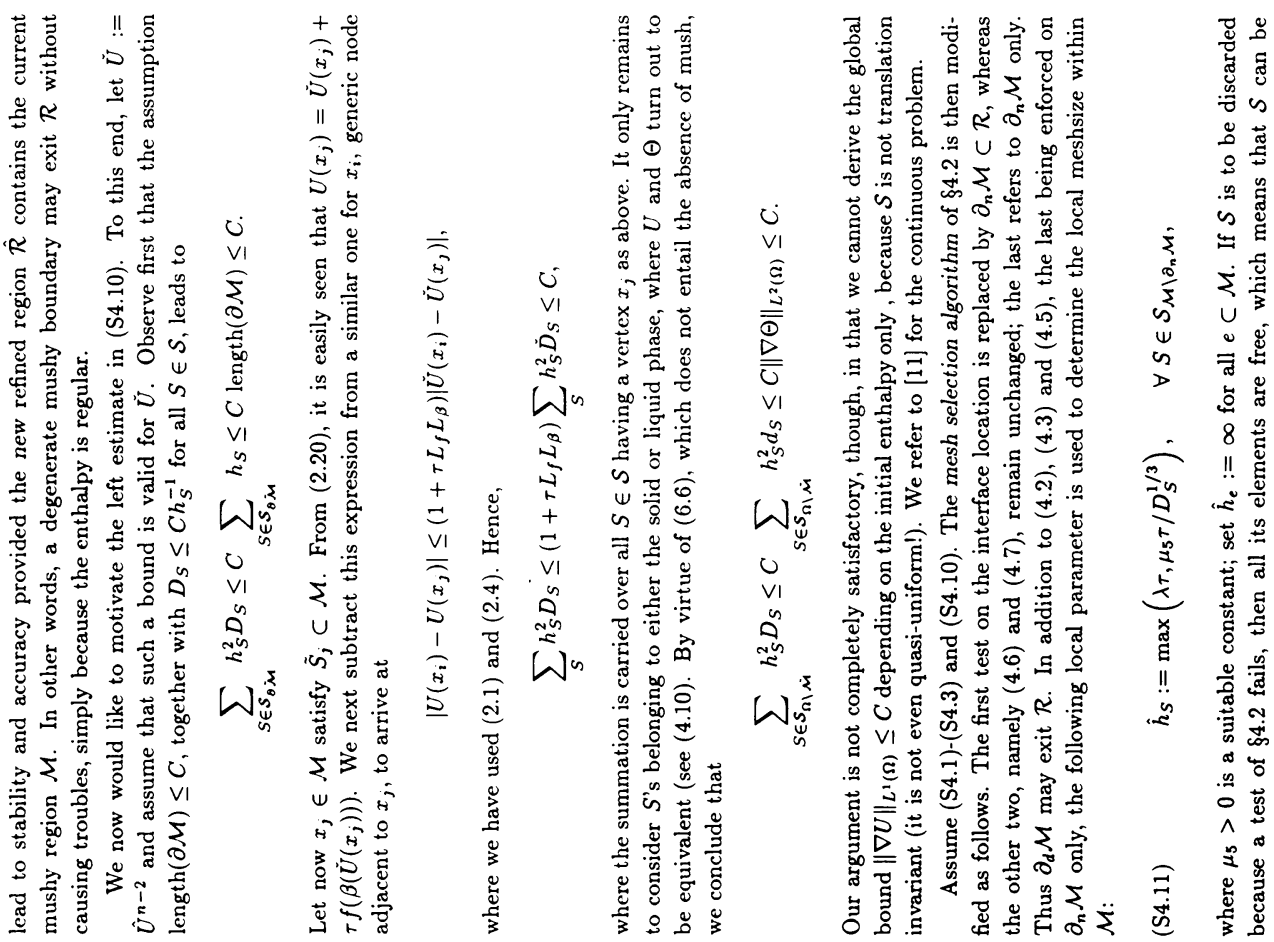




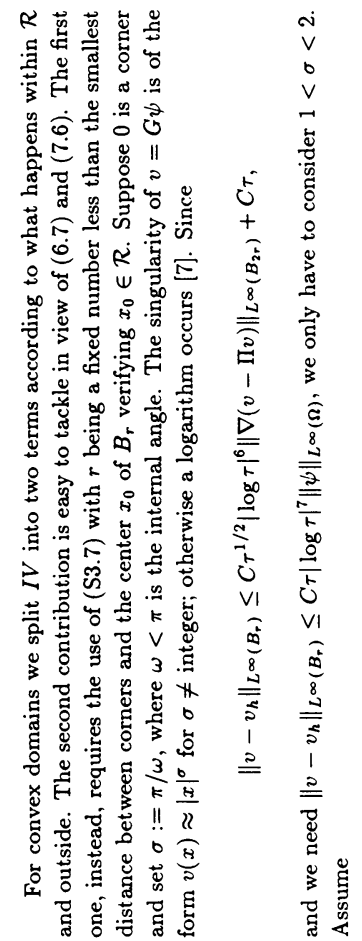


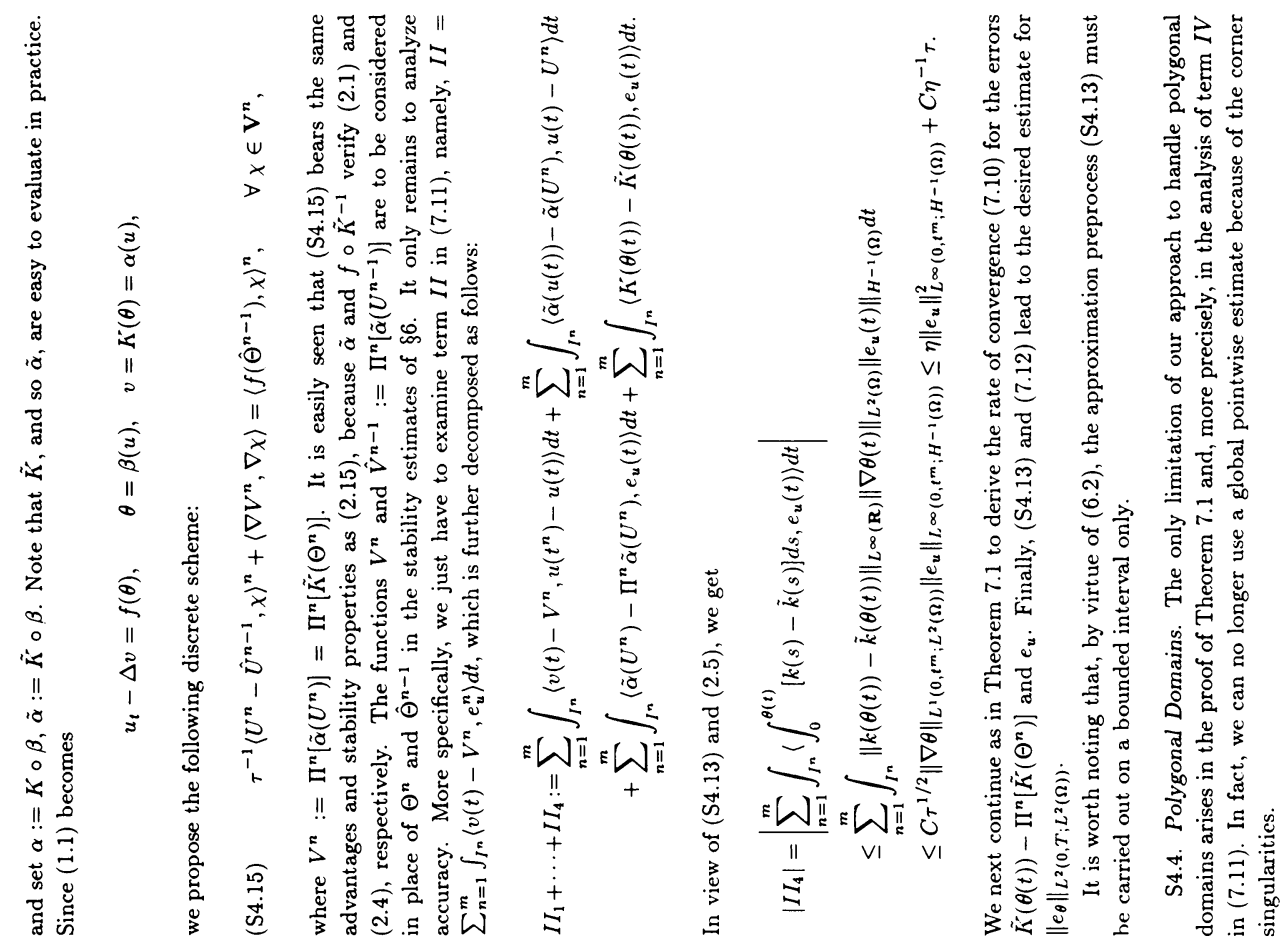